Article Type: Letter

2

\section{Application of a novel haplotype-based scan for local adaptation to study high-altitude adaptation in rhesus macaques}

\author{
Zachary A. Szpiech ${ }^{\dagger}$ \\ szpiech@psu.edu
}

Department of Biology

Pennsylvania State University

University Park, PA 16801 USA

Institute for Computational and Data Sciences

Pennsylvania State University

University Park, PA 16801 USA

Department of Biological Sciences

Auburn University

Auburn, AL 36842, USA

Taylor E. Novak*

tep0007@auburn.edu

Department of Biological Sciences

Auburn University

Auburn, AL 36842, USA

Nick P. Bailey*

npb0015@auburn.edu

Department of Biological Sciences

Auburn University

Auburn, AL 36842, USA

Laurie S. Stevison ${ }^{\dagger}$

Iss0021@auburn.edu

Department of Biological Sciences

Auburn University

Auburn, AL 36842, USA

†Correspondence: szpiech@psu.edu, Iss0021@auburn.edu

${ }^{*}$ Equal Contribution

Running Title: High-altitude adaptation in rhesus macaques

Keywords: Adaptation, genome-scan, high-altitude, EGLN1, selection, macaque

Abstract Word Count (300 max): 214

Total Word Count (5000 max): 5149 


\section{Abstract}

When natural populations split and migrate to different environments, they may experience different selection pressures that can lead to local adaptation. To capture

54 the genomic patterns of a local selective sweep, we develop XP-nSL, a genomic scan

55 for local adaptation that compares haplotype patterns between two populations. We

56 show that XP-nSL has power to detect ongoing and recently completed hard and soft

57 sweeps, and we then apply this statistic to search for evidence of adaptation to high

58 altitude in rhesus macaques. We analyze the whole genomes of 23 wild rhesus

59 macaques captured at high altitude (mean altitude $>4000 \mathrm{~m}$ above sea level) to 22 wild

60 rhesus macaques captured at low altitude (mean altitude $<500 \mathrm{~m}$ above sea level) and

61 find evidence of local adaptation in the high-altitude population at or near 303 known

62 genes and several unannotated regions. We find the strongest signal for adaptation at

63 EGLN1, a classic target for convergent evolution in several species living in low oxygen

64 environments. Furthermore, many of the 303 genes are involved in processes related to

65 hypoxia, regulation of ROS, DNA damage repair, synaptic signaling, and metabolism.

66 These results suggest that, beyond adapting via a beneficial mutation in one single

67 gene, adaptation to high altitude in rhesus macaques is polygenic and spread across

68 numerous important biological systems.

\section{Impact Summary}

When positive selection is ongoing or a beneficial mutation has recently fixed in a

71 population, genetic diversity is reduced in the vicinity of the adaptive allele, and we

72 expect to observe long homozygous haplotypes at high frequency. Here we develop a

73 statistic that summarizes these expected patterns and compares between two 
74 populations in order to search for evidence of adaptation that may have occurred in one

75 but not the other. We implement this statistic in a popular and easy-to-use software

76 package, and then apply it to study adaptation to high altitude in rhesus macaques.

77 Extreme environments pose a challenge to life on multiple fronts. Very high-

78 altitude environments are one such example, with low atmospheric oxygen, increased

79 ultraviolet light exposure, harsh temperatures, and reduced nutrition availability. In spite

80 of these challenges, many plants and animals, including humans, have genetically

81 adapted to cope with these hardships. Here we study two populations of rhesus

82 macaques, one living at high altitude and one living close to sea level. We apply our

83 novel statistic to compare their haplotype patterns between them to search for evidence

84 of genetic changes that are indicative of adaptation to their environment.

We find evidence for adaptation at a critical gene that helps control physiological

86 response to low-oxygen, one that has been the target of repeated convergent evolution

87 across many species. We also find evidence for positive selection across a range of

88 traits, including metabolic and neurological. This work helps to explain the evolutionary

89 history of the rhesus macaque and furthers our understanding about the ways

90 organisms genetically adapt to high-altitude environments.

\section{Introduction}

92 Selective sweeps produce regions of reduced genetic diversity in the vicinity of

93 an adaptive mutation. These patterns manifest as long extended regions of

94 homozygous haplotypes segregating at high frequency (Przeworski 2002; Sabeti et al.

95 2002; Kim and Nielsen 2004; Garud et al. 2015). In the event of a de novo mutation that

96 is adaptive in a population, we expect the haplotype it resides on to rapidly rise in 
97 frequency in the population (called a 'hard' sweep). On the other hand, if an ancestrally

98 segregating neutral or mildly deleterious allele turned out to be adaptive in a new

99 environment, it would likely reside on two or more haplotypes, which would rapidly rise

100 in frequency in the population (called a 'soft' sweep) (Hermisson and Pennings 2005;

101 Pennings and Hermisson 2006). As both of these processes happen on a time scale

102 faster than mutation or recombination can act to break up the sweeping haplotypes, we

103 expect to observe long and low diversity haplotypes at high frequency in the vicinity of

104 an adaptive mutation. However, if this mutation either does not exist or is not adaptive in

105 a sister population, we would not expect a sweep to occur and thus we would not

106 expect to observe similar haplotype patterns.

107 To capture these haplotype patterns and contrast them between a pair of

108 populations, we develop XP-nSL, a haplotype-based statistic with good power to detect

109 partial, fixed, and recently completed hard and soft sweeps by comparing a pair of

110 populations. XP-nSL is an extension of nSL (Ferrer-Admetlla et al. 2014) and does not

111 require a genetic recombination map for computation. The lack of dependence on a

112 recombination map is important, as other statistics for identifying positive selection are

113 biased towards low-recombination regions (O'reilly et al. 2008), but the approach taken

114 by nSL has been shown to be more robust (Ferrer-Admetlla et al. 2014). Both nSL and

115 XP-nSL summarize haplotype diversity by computing the mean number of sites in a

116 region that are identical-by-state across all pairs of haplotypes. Whereas nSL contrasts

117 between haplotype sets carrying an ancestral or a derived allele in a single population,

118 XP-nSL contrasts between haplotype sets in two different populations, allowing it to

119 detect local adaptation. 
An extreme example of adaptation to a local environment is the transition to high-

121 altitude living. Organisms living at high altitude are confronted with many challenges,

122 including a low-oxygen atmosphere and increased ultraviolet light exposure, and these

123 harsh environments inevitably exert strong selection pressure. Indeed, adaptation to

124 high-altitude living has been studied extensively across many organisms from plants,

125 including monocots (Gonzalo-Turpin and Hazard 2009; Ahmad et al. 2016) and dicots

126 (Kim and Donohue 2013; Liu et al. 2014; Munne-Bosch et al. 2016; Guo et al. 2018), to

127 numerous animals including amphibians (Yang et al. 2016), canids (Li et al. 2014; Wang

128 et al. 2014; Wang et al. 2020), humans (Bigham et al. 2009; Bigham et al. 2010; Xu et

129 al. 2010; Yi et al. 2010; Peng et al. 2011; Huerta-Sanchez et al. 2013; Huerta-Sanchez

130 et al. 2014; Jeong et al. 2014), yaks (Qiu et al. 2012), birds (Cai et al. 2013; Qu et al.

131 2013; Wang et al. 2015; Graham and Mccracken 2019), boars (Li et al. 2013), mice

132 (Storz et al. 2007; Cheviron et al. 2012; Schweizer et al. 2019; Storz et al. 2019; Velotta

133 et al. 2020), moles (Campbell et al. 2010), antelope (Ge et al. 2013), and horses

134 (Hendrickson 2013). Liu et al. (2018) recently sequenced and published the whole

135 genomes of 79 wild-born Chinese rhesus macaques collected from multiple sites in

136 China. Among these animals, 23 were sampled from far western Sichuan province in a

137 region with mean altitude $>4000 \mathrm{~m}$ above sea level (Liu et al. 2018), providing an

138 opportunity to study the genetics of local adaption to high altitude in rhesus macaques.

139 Rhesus macaques are the second most widely distributed primate, with a range

140 extending from Afghanistan to Vietnam and from a latitude of 15 to 38 degrees north

141 (Fooden 2000). Early ancestors of the macaque migrated out of Africa to the Eurasian

142 continent approximately 7 mya-the earliest catarrhine fossils on the continent are 
143 macaque-like (Stewart and Disotell 1998). Modern rhesus macaques trace a recent

144 origin to Southeast Asia, with a major migratory split occurring approximately 162 kya

145 separating the ancestors of modern Indian and Chinese rhesus macaques (Hernandez

146 et al. 2007). Macaques have proven to be quite evolutionarily successful, demonstrating

147 ecological flexibility and adaptability via developmental plasticity and behavioral

148 changes (Richard et al. 1989; Madrid et al. 2018). Other studies have looked at how the

149 rhesus macaque radiation has led to population-level adaptation to climate and food

150 availability (Liu et al. 2018).

$151 \quad$ Here we test and evaluate our XP-nSL statistic and apply it to study the genomic

152 consequences of high-altitude living in the rhesus macaque. We use it to compare the

153 haplotype patterns of the 23 animals from the high-altitude population with another 22

154 that Liu et al. (2018) sampled in lower-lying regions in eastern China with a mean

155 altitude $<500 \mathrm{~m}$ above sea level.

156 Methods

157 Data Preparation

$158 \quad$ Liu et al. (2018) generated whole genome sequencing data for 79 Chinese

159 macaques and called all biallelic polymorphic sites according to GATK best practices

160 using rheMac8, identifying 52,534,348 passing polymorphic autosomal sites. We then

161 filter all loci with $>10 \%$ missing data leaving $35,639,395$ biallelic sites. Next, the

162 program SHAPEIT v4.1.2 (Delaneau et al. 2019) was used to phase haplotypes in the

163 full data set with a genetic map that was available for rheMac8 (Bcm-Hgsc 2020).

164 SHAPEIT performs imputation during phasing for any missing genotypes. Our analyses

165 here focus on 45 of the 79 samples from Liu et al. (2018), representing 23 from high- 
166 altitude regions of China (M. $m$. lasiotis) and 22 from low-altitude regions of China (M.

167 m. littoralis), based on capture location information. See Table S1 for individual IDs

168 used.

Liu et al. (2018) also inferred joint demographic histories for their five

170 populations, and we extract the demographic parameters for our two of interest. This

171 demographic history is recapitulated in Fig. 1 with detailed parameters given in Table 1,

172 which are then used for simulations to test XP-nSL.

173 A Statistic for Detecting Local Adaptation

We developed a cross-population haplotype-based statistic, XP-nSL, to scan for

175 regions of the genome implicated in local adaptation between two populations by

176 extending nSL (Ferrer-Admetlla et al. 2014), each of which is defined below.

178 carrying the ancestral or derived allele, respectively, and let $n_{A}(k)=|A(k)|$ and

$n_{D}(k)=|D(k)|$. Next $L_{i j}(k)$ is defined as the number of consecutive sites at which

181 at site $k$ is $n S_{L}(k)=\log \frac{S L_{A}(k)}{S L_{D}(k)}$, where $S L_{A}(k)=\left(\begin{array}{c}n_{A}(k) \\ 2\end{array}\right)^{-1} \sum_{i<j \in A(k)} L_{i j}(k)$ and $S L_{D}(k)=$

$182\left(\begin{array}{c}n_{D}(k) \\ 2\end{array}\right)^{-1} \sum_{i<j \in D(k)} L_{i j}(k) . S L_{A}(k)$ and $S L_{D}(k)$ represent the mean $L_{i j}(k)$ over all pairs of

183 haplotypes carrying either the ancestral or derived allele at locus k, respectively. nSL

184 scores are then normalized genome-wide in site-frequency bins either with respect to

185 the empirical background or neutral simulations with a matching demographic history.

186 The nSL computation is illustrated in Ferrer-Admetlla et al. (2014). nSL is implemented

187 in nsl (Ferrer-Admetlla et al. 2014) and selscan v1.1.0+ (Szpiech and Hernandez 188 2014). 
$\mathrm{XP}-\mathrm{nSL}$ is defined similarly, except instead of comparing sets of haplotypes containing ancestral or derived alleles, it compares sets of haplotypes between two

191 different populations. Let $P_{1}(k)$ and $P_{2}(k)$, represent the set of haplotypes at site $k$ in

192 population 1 and population 2 , respectively, and let $n_{P_{1}}(k)=\left|P_{1}(k)\right|$ and $n_{P_{2}}(k)=$

$193\left|P_{2}(k)\right|$. Then XP-nSL at site $k$ is $X P n S_{L}(k)=\log \frac{S L_{P_{1}}(k)}{S L_{P_{2}}(k)}$, where $S L_{P_{1}}(k)=$

$\left(\begin{array}{c}n_{P_{1}}(k) \\ 2\end{array}\right)^{-1} \sum_{i<j \in P_{1}(k)} L_{i j}(k)$ and $S L_{P_{2}}(k)=\left(\begin{array}{c}n_{P_{2}}(k) \\ 2\end{array}\right)^{-1} \sum_{i<j \in P_{2}(k)} L_{i j}(k) . S L_{P_{1}}(k)$ and $S L_{P_{2}}(k)$

195 represent the mean $L_{i j}(k)$ over all pairs of haplotypes in population 1 or population 2 at

196 locus k, respectively. XP-nSL scores are then normalized genome-wide either with

197 respect to the empirical background or neutral simulations with a matching demographic

198 history. The XP-nSL computation is illustrated in Fig. 2 with a toy example. We

199 implement XP-nSL in selscan v1.3.0+ (Szpiech and Hernandez 2014) to facilitate wide

200 adoption. It is worth noting that XP-nSL is analogous to XP-EHH (Sabeti et al. 2007) as

$201 \mathrm{nSL}$ is analogous to iHS (Voight et al. 2006).

The goal of these statistics is to capture a signal of extended regions of low

203 diversity on sweeping haplotypes (emblematic of an ongoing or recently completed

204 selective sweep) within a population (nSL) or on sweeping haplotypes in one population

205 versus another (XP-nSL). When XP-nSL scores are positive this suggests evidence for

206 a hard or soft sweep in population 1, and when XP-nSL scores are negative this

207 suggests evidence for a hard or soft sweep in population 2.

\section{Simulations}

In order to test the ability of XP-nSL to detect ongoing and recently completed

210 hard and soft sweeps, coalescent simulations were performed conditional on an allele

211 frequency trajectory with the program discoal (Kern and Schrider 2016). discoal 
212 simulates an allele frequency trajectory for a single non-neutral allele backwards in time

213 and then simulates a neutral coalescent process conditional on this trajectory. This

214 takes advantage of the speed and efficiency of the coalescent while still being able

215 simulate genetic diversity patterns in the vicinity of a non-neutral locus.

216 All simulations were run with a two-population divergence demographic history

217 (Fig. 1 and Table 1), as inferred by Liu et al. (2018), and given by the following

218 discoal command line arguments -p 244644 -en $0 \quad 1 \quad 0.230410476572876$ -

219 en 0.07196466627544210 .278345739259351 -en 0.0865583593291531

2201.282885999320505 -ed 0.14621490564289510 -en

$2210.146214905642895 \quad 0 \quad 4.089940389782871$. Here 46 haplotypes were sampled

222 from population 0 , which has the demographic history of the high-altitude population, 223 and 44 haplotypes were sampled from population 1, which has the demographic history

224 of the low-altitude population. A mutation rate of $\mu=2.5 \times 10^{-8}$ (Fan et al. 2018) was

225 used, along with a recombination rate of $r=5.126 \times 10^{-9}$, which was computed as the

226 genome-wide mean rate from the rheMac8 recombination map (Bcm-Hgsc 2020). A 500

227 kb region was simulated, thus giving a scaled mutation rate and scaled recombination

228 parameters for discoal as -t 809.425 -r $165.967 .5,349$ replicates of neutral

229 sequence were simulated under this model, representing approximately the entire

230 macaque genome minus $500 \mathrm{~kb}$. Thus, the total simulated length of all neutral regions

231 plus one selected region is approximately equal to the macaque genome length.

232 For non-neutral simulations, sweep scenarios were simulated with a positive

233 additive selection coefficient $s \in\{0.01,0.02,0.05\}$, which is provided to discoal as a

234 scaled selection coefficient $2 N_{1} s \in\{323.77,647.54,1618.85\}$ (discoal flag -a). Soft 
235 sweeps are simulated as a mutation that arose neutral and turned beneficial at a

236 particular establishment frequency $e \in\{0.01,0.02,0.03,0.04,0.05,0.10,0.20\}$ (discoal flag

$237-f)$. Hard sweeps are simulated from a de novo mutation that was never neutral, i.e.

$238 e=0$. Finally, sweeps were conditioned on having either reached a certain frequency in

239 the population at the time of sampling, $f \in\{0.7,0.8,0.9,1.0\}$ (discoal flag $-c$ ), or that

240 the adaptive mutation reached fixation some number of generations prior to sampling,

$241 g \in\{50,100,200\}$, which is provided to discoal in coalescent units $g / 2 N_{1} \in$

$242\left\{1.5443 \times 10^{-3}, 3.0886 \times 10^{-3}, 6.1774 \times 10^{-3}\right\}$ (discoal flag $\left.-w s\right)$. For the sake of

243 being conservative in our estimation of power (Voight et al. 2006; Sabeti et al. 2007), it

244 was assumed the actual adaptive mutation remains unsampled (discoal flag $-\mathrm{h}$ ) even

245 though whole genome sequencing data are being analyzed. For each combination of

246 parameter values, 500 replicates were simulated.

247 Neutral Simulations of Mismatched Histories

248 We also generate neutral simulations for three mismatched demographic

249 histories, in order to study how a mismatched demographic history may influence the

250 power and false positive rates of XP-nSL if used as a normalization baseline. We name

251 these mismatched histories "Rand", "Under", and "Over" and generate 5,349 replicates

252 for each one.

253 For the "Rand" history, each parameter is (uniformly) randomly chosen from

254 within the $95 \% \mathrm{Cl}$ as inferred by (Liu et al. 2018) (see Table 1). The parameters are

255 resampled for each replicate of the "Rand" history, and the condition

$256 T_{1}<T_{2}<T_{3}$ is enforced. For the "Under" history, the present-day population size

257 parameters $N_{1}$ and $N_{2}$ are the only ones modified. They are set to $N_{1}=8255$ at the 
258 extreme low end of the $95 \% \mathrm{Cl}$ and $N_{2}=5006$ at the extreme high end of the $95 \% \mathrm{Cl}$.

259 This represents a scenario where the difference in population sizes is underestimated.

260 For the "Over" history, once again the present-day population size parameters N1 and

261 N2 are the only ones modified. They are set to $N_{1}=26825$ at the extreme high end of

262 the $95 \% \mathrm{Cl}$ and $N_{2}=660$ at the extreme low end of the $95 \% \mathrm{Cl}$. This represents a

263 scenario where the difference in population sizes is overestimated.

264 Detecting Local Adaptation in Real Data

265 From the phased data set, animals captured at high altitude $(n=23)$ and animals

266 captured at low altitude $(n=22)$ were subset (see Table S1). Using selscan v1.3.0

267 (Szpiech and Hernandez 2014) to compute raw XP-nSL scores across the genome

268 (selscan flags -xpnsl -vcf high-altitude.vcf-vcf-ref low-altitude.vcf), scores were then

269 normalized using the genome-wide empirical background with selscan's norm v1.3.0

270 program (norm flag --xpnsl). The low-altitude population was used as the reference

271 population, so positive XP-nSL scores correspond to long homozygous haplotypes and

272 a possible sweep in the high-altitude population compared to the low-altitude

273 population, and vice versa for negative XP-nSL scores. A Manhattan plot of genome-

274 wide normalized XP-nSL scores $>2$ is plotted in Fig. S7.

275 In order to identify regions implicated as potentially adaptive, we search for

276 clusters of extreme scores along a chromosome. Using selscan's companion program

277 norm $\mathrm{v} 1.3 .0$, the genome is divided into non-overlapping $100 \mathrm{~kb}$ regions and both the

278 maximum XP-nSL score and the fraction of XP-nSL scores $>2$ are computed (norm

279 flags -xpnsl -bp-win -winsize 100000). norm then creates 10 quantile bins (--qbins 10)

280 for windows with more than 10 sites per window (--min-snps 10) and identifies the top 
$2811 \%$ of windows with the highest fraction of extreme scores (Fig. S6 and Table S4). Each

282 window is then annotated with the ensembl rheMac8 gene list, and a maximum XP-nSL

283 score is assigned to a given gene based on the max-score in the $100 \mathrm{~kb}$ window with

284 which it overlaps. If a gene overlaps more than one $100 \mathrm{~kb}$ window, it is assigned the

285 top max-score from among the windows.

\section{Results}

287 Power Analysis of XP-nSL

First, we evaluate the performance of XP-nSL based on simulations. After

289 computing XP-nSL for all sites in all simulations, scores were normalized by subtracting

290 the mean and dividing by standard deviation of the neutral simulations, giving the

291 neutral scores an approximately $\mathrm{N}(0,1)$ distribution (Fig. S1).

We consider the maximum score in a $100 \mathrm{~kb}$ interval as way to identify regions

293 under positive selection similar to Voight et al. (2006). To get the null distribution of

294 max-scores, the maximum score is computed in the central $100 \mathrm{~kb}$ of each neutral

295 simulation. The distribution of max-scores in neutral simulations had a median of 2.093

296 with $95 \%$ of the mass between 0.804 and 3.492, which is represented in Fig. 3 as a

297 solid horizontal black line (median) and two dashed horizontal black lines (95\% interval).

298 Next, the maximum score is computed in the central $100 \mathrm{~kb}$ of all non-neutral

299 simulations, and the median and 95\% intervals are plotted for each parameter

300 combination. Fig. 3 shows good separation between the neutral distribution of max-

301 scores and the distribution of max-scores for a range of non-neutral parameters,

302 suggesting that our statistic can distinguish between neutral and non-neutral scenarios.

303 Note that soft sweeps that start at 0.1 or 0.2 frequency see the least separation from the 
304 neutral distribution. To evaluate the power of the max-score statistic, the $99^{\text {th }}$ percentile

305 of the max-score distribution is computed in the neutral distribution (neutral ${ }_{99}=3.792$ )

306 and power is calculated as the mass of each non-neutral max score distribution

$307>$ neutral $_{99}$. With this approach, even if the entire genome is neutrally evolving, at most

$3081 \%$ of the genome will be identified as putatively under selection thus fixing the false

309 positive rate at $1 \%$ at most. Results are plotted in Fig. 4A, which shows good power to

310 detect both incomplete and completed sweeps. For soft sweeps that start at a frequency

$311 \leq 0.03$, power is $>75 \%$ when the sweep is near or just past fixation; the ability to

312 detect soft sweeps falls off for sweeps that start $>0.03$ frequency.

313 Next, we consider that due to linkage disequilibrium consecutive scores will be

314 correlated, and we should therefore expect clusters of extreme scores in true non-

315 neutral regions. We thus consider a window-based approach to identify selected regions

316 similar to Voight et al. (2006), where the top $1 \%$ windows with a high number of extreme

317 scores are identified. Taking the central $100 \mathrm{~kb}$ region of each simulation, the fraction of

318 XP-nSL scores $>2$ (representing approximately the highest $2 \%$ of all neutral scores) is

319 computed. Since each $100 \mathrm{~kb}$ window has a variable number of sites within it, windows

320 with fewer sites are more likely to have a higher fraction of extreme scores by chance.

321 Thus, windows are binned by number of sites into 10 quantile bins, and the top $1 \%$ of

322 windows with the highest fraction of extreme scores in each bin establishes the

323 threshold beyond which a window is taken as putatively selected, as in Voight et al.

324 (2006). With this approach, even if the entire genome is neutrally evolving, at most $1 \%$

325 of the genome will be identified as putatively under selection thus fixing the false

326 positive rate at $1 \%$ at most. Power is computed for each non-neutral parameter set as 
327 the proportion of replicates for which the central $100 \mathrm{~kb}$ exceeds the $1 \%$ threshold as

328 calculated from neutral simulations. The results are plotted in Fig. 4B, which shows

329 improved power over the max-score approach across a wider range of parameters.

330 Indeed, using the window-based method, power to detect soft sweeps improves

331 substantially across the parameter space, with $>75 \%$ power to detect soft sweeps at or

332 near fixation that started at frequency $\leq 0.05$.

333 We next consider how XP-nSL power compares to nSL, XP-EHH, and Fst. We

334 compare to nSL since XP-nSL is an extension of it, to XP-EHH as it is a similar

335 haplotype-based two-population selection statistic, and to Fst as it a popular two-

336 population method used to infer local adaptation. For all simulations, nSL and XP-EHH

337 are computed using selscan v1.3.0 (Szpiech and Hernandez 2014). Normalization,

338 identification of top windows, and power calculation was done as described above for

339 XP-nSL. Fst is computed using VCFtools v0.1.16 (Danecek et al. 2011), which

340 implements Weir and Cockerham's formulation (Weir and Cockerham 1984), in 100kb

341 windows for all simulations. The $99^{\text {th }}$ percentile $F_{S T}$ value was determined from the

342 central $100 \mathrm{~kb}$ window among all neutral simulations. Power was then computed for

343 each non-neutral parameter set as the proportion of replicates for which the FsT value of

344 the central $100 \mathrm{~kb}$ window is greater than the neutral threshold as calculated from

345 neutral simulations.

346 Fig. 5 shows the difference in power between XP-nSL and each statistic (raw

347 power for nSL, XP-EHH, and FST shown in Fig. S4) over the simulated parameter

348 space, where positive values indicate that XP-nSL has more power than the comparison

349 statistic. XP-nSL is compared to nSL in Fig. 5A, which shows XP-nSL improves on nSL 
350 across nearly the entire parameter space and especially for sweeps that have fixed in

351 the recent past. XP-nSL is compared to XP-EHH in Fig. 5B, which shows XP-nSL

352 improving on $\mathrm{XP}-\mathrm{EHH}$ for soft sweeps $(e>0)$. Finally, $\mathrm{XP}-\mathrm{nSL}$ is compared to $\mathrm{F}_{\mathrm{ST}}$ in

353 Fig. 5C, which shows XP-nSL improving on Fst mostly for incomplete sweeps, although

354 Fst $_{\mathrm{St}}$ performed better post-fixation for certain soft sweep scenarios $(e \geq 0.05)$.

\section{Caveats for Using Neutral Simulations to Normalize Real Data}

In principle, one could use matched neutral simulations as a normalization

357 baseline when analyzing real data. However, we can only recommend this approach

358 when the populations being studied have very well characterized (1) joint demographic

359 histories, (2) mutation rates, (3) and recombination rates, as a mismatch can skew the

360 power and false positive rates of the statistic. To illustrate this point, we generated three

361 mismatched sets of neutral simulations "Rand", "Under", and "Over" (see Methods) to

362 use as a normalization baseline for our original simulations.

When neutral simulations are normalized with the correct demographic history,

364 they approximately follow a standard normal distribution (Fig. S1 and Fig. S2), however

365 the distribution of neutral scores gets badly distorted when one of the mismatched

366 histories is used (Fig. S2). These distortions have practical consequences for making

367 inferences. Power was calculated for XP-nSL using the window-based method

368 described above but using each mismatched history as a normalization baseline (Fig.

369 S3). The false positive rate for each scenario was also estimated by calculating the

370 proportion of neutral simulations that are identified as under selection for each scenario

371 (Table S2). Fig. S3A-B shows that for the "Rand" and "Under" normalization scenarios,

372 power was greatly reduced across the whole parameter space. Only hard sweeps with 
373 the strongest selection coefficients post-fixation were likely to be identified. False

374 positive rates for these scenarios were at 0 (Table S3), whereas for a matched history it

375 was estimated at $9.908 \times 10^{-3}$. Fig.S3C shows that, for the "Over" normalization

376 scenario, power was uniformly excellent, however when analyzing neutral simulations,

377 the false positive rate was estimated at 0.9538 . This suggests that using such a badly

378 matched demographic history for normalization creates a near complete inability to

379 distinguish between neutral and selected regions.

380 Identifying Evidence for Adaptation to High Altitude in Rhesus Macaques

$381 \quad$ Next, we analyzed the pair of rhesus macaque populations using XP-nSL,

382 searching for evidence of local adaptation in the high-altitude population. Using the low-

383 altitude population as the reference population, normalized $X P n S_{L}>0$ corresponds to

384 longer and higher frequency haplotypes in the high-altitude population, with very large

385 positive scores and clusters of large scores suggesting evidence for positive selection.

386 Dividing the genome into $100 \mathrm{~kb}$ windows, the maximum XP-nSL score of that region is

387 assigned to each gene in it (see Methods), thus multiple genes may have the same

388 max-score by virtue of being in the same $100 \mathrm{~kb}$ window. Genes overlapping multiple

$389100 \mathrm{~kb}$ windows were assigned the top max-score among the windows.

390 Using the per-gene max-scores, PANTHER (Mi et al. 2019) gene ontology

391 categories were tested for enrichment of high scores, where significance suggests an

392 enrichment of signals of positive selection among genes involved. Significant terms

393 related to regulation of ion transport and synaptic signaling (Table 2), each of which are

394 affected by hypoxic conditions (Karle et al. 2004; Corcoran and O'connor 2013). 
From the genomic regions that were identified to contain a high proportion of extreme positive scores (see Methods), 303 annotated genes were found across 270 regions. A permutation test (10,000 replicates) that randomly shuffles $270100 \mathrm{~kb}$ regions around the genome indicates that this is substantially fewer than one would expect by chance $\left(p=1.4 \times 10^{-3} ;\right.$ Fig. S5), indicating that the method is not simply

400 randomly picking gene regions. These regions, their characteristics, and the genes 401 contained therein are given in Table S2. A PANTHER (Mi et al. 2019) gene ontology

402 overrepresentation test indicates a 9.04-fold enrichment of genes associated with 403 monooxygenase activity $\left(F D R=4.47 \times 10^{-2}\right)$.

The monooxygenases in the selected regions include FMO2, FMO5, CYP2C8, CYP2C9, CYP2C93, and ENSMMUT00000011129. These genes are important for the metabolism of oxygen and the generation of reactive oxygen species (ROS) (Krueger 407 and Williams 2005). Under the physiological stress of a low-oxygen environment, ROS 408 levels increase and cause oxidative damage, and, in humans, long-term adaptation to 409 high altitudes includes adaptation to oxidative damage (Janocha et al. 2017). Indeed, 410 AOX1 is also identified in our top regions, mutations in which have been shown to affect 411 ROS levels in humans (Foti et al. 2017).

412 The genome-wide top ten $100 \mathrm{~kb}$ windows based on the percentage of extreme

413 XP-nSL scores are summarized in Table 3, and these windows overlap several genes, 414 including EGLN1. The EGLN1 locus is directly adjacent to the single strongest selection 415 signal identified in the entire genome (Fig. 6). This region has the third highest cluster of 416 extreme scores (Table 3), contains the highest XP-nSL score in the entire genome $417\left(\mathrm{chr1}: 207,698,003, X P n S_{L}=6.54809\right)$, and contains six of the top ten genome wide XP- 
$418 \mathrm{nSL}$ scores (colored dark red in Fig. 6). EGLN1 is a regulator of oxygen homeostasis

419 (To and Huang 2005) and is a classic target for adaptation to low-oxygen levels, having

420 repeatedly been the target of adaptation in numerous organisms living at high altitude

421 around the world (Bigham et al. 2009; Bigham et al. 2010; Jeong et al. 2014; Graham

422 and Mccracken 2019). In addition to EGLN1, other genes related to lung function,

423 oxygen use, and angiogenesis had evidence for local adaptation between low- and

424 high-altitude populations: TRPM7, RBPJ, and ENSMMUT00000040566 (Table S2).

425 TRPM7 downregulation in a hypoxia-induced rat model was associated with pulmonary

426 hypertension (PAH) (Xing et al. 2019). ENSMMUT00000040566 is a MAPK6 ortholog,

427 which interacts with EGLN3 (Rodriguez et al. 2016), and both it and RBPJ are involved

428 in angiogenesis (Ramasamy et al. 2014).

429 Due to the reduced oxygen levels at high altitudes, we expect genes involved in

430 metabolism and respiration may be under positive selection. Indeed, MDH1 encodes a

431 critical enzyme in the citrate cycle (Tanaka et al. 1996) and is found in the top ten

432 genome-wide regions (Table 3). A paralog of MDH1, MDH1B, has been previously

433 identified as a target of selection in humans living at high altitude (Yi et al. 2010).

434 ACADM and COX15 are also found in putatively adaptive regions (Table S2). Mutations

435 in and differing expression levels of ACADM are related to oxidative stress and

436 mitochondrial dysfunction in human disease (Xu et al. 2018). COX15 is involved in

437 oxidative phosphorylation (Alston et al. 2017), and cytochrome c oxidase (COX) genes

438 have previously been identified as under selection in primates relative to other

439 mammals (Osada and Akashi 2012). 
High-altitude environments present a particular metabolic challenge to organisms

441 that must maintain a stable internal body temperature (Rosenmann and Morrison 1974;

442 Hayes and Chappell 1986; Chappell and Hammond 2004), a result of increased oxygen

443 demand from aerobic thermogenesis conflicting with lower oxygen availability. It has

444 been shown that highland deer mice have adapted by increased capacity to metabolize

445 lipids compared to lowland deer mice (Cheviron et al. 2012), and previous studies in

446 rhesus macaques have shown there may be drastic differences in diets between high-

447 and low-altitude populations (Zhao 2018). Indeed, in the high-altitude macaque

448 population studied here, genes related to lipid and fat metabolism (DOCK7,

449 ST6GALNAC5, ANGPTL3, and ACACA) were found in putative adaptive regions.

450 Across human populations, these genes are all responsible for varying blood levels of

451 fatty acids (Guo et al. 2016; Dewey et al. 2017; Hebbar et al. 2018). Furthermore,

452 ACACA has been shown to vary fatty acid blood concentrations and be differentially

453 expressed in highland versus lowland swine populations (Shang et al. 2019).

455 vesicular trafficking and neurotransmitter release (Kumar et al. 2015). As primate brains

456 use large amounts of oxygen and energy to function (Osada and Akashi 2012)

457 signatures of selection on neurological genes may be expected across populations

458 living at altitudes with differing oxygen levels. In addition to STXBP5L, several genes

459 related to neural development and synaptic formation (JAG2, TRPM7, DOCK7, NSG2,

460 AUTS2) were identified (Table S2). JAG2 is involved in the Notch signaling pathway.

461 While Notch signaling is involved in many developmental and homeostatic processes,

462 its role in neuronal differentiation in the mammalian brain is notable in this context 
463 (Cardenas et al. 2018). TRPM7, in addition to its association with PAH, plays a role in

464 hypoxic neuronal cell death (Aarts et al. 2003).

465 DNA damage, including double strand breaks and pyrimidine dimerization, can

466 manifest as a result of oxidative stress (Ye et al. 2016) or increased exposure to UV

467 radiation (Zhang et al. 2000; Greinert et al. 2012), both of which increase at high

468 altitudes. Three DNA damage repair genes are in our set of genes identified in positively

469 selected regions. The ring finger protein RNF138 is in our list of top ten genomic

470 windows (Table 3) and has been shown to promote DNA double-strand break repair

471 (Ismail et al. 2015). Furthermore, the DNA polymerase POLH also appears in a

472 putatively adaptive region (Table S2) and is known to be able to efficiently bypass

473 pyrimidine dimer lesions (Zhang et al. 2000). Finally, PAXIP1 appears in Table S2 as

474 well and has been shown to promote repair of double strand breaks through

475 homologous recombination (Wang et al. 2010).

Interestingly, within the putatively selected region that includes PAXIP1 is an

477 uncharacterized long non-coding RNA (IncRNA), ENSMMUT00000081951, that was

478 recently annotated in the rheMac10 genome build. This IncRNA has high sequence

479 similarity to a IncRNA on the same synteny block in humans called PAXIP1-AS1. In

480 human pulmonary artery smooth muscle cells, knockdown of PAXIP1-AS1 leads to an

481 abnormal response to $\mathrm{PAH}$ where migration and proliferation of cells is reduced, and

482 overexpression of PAXIP-AS1 leads to apoptosis resistance (Jandl et al. 2019).

483 Although, we note that any link between PAH and ENSMMUT00000081951 in rhesus

484 macaques is highly speculative at this point.

\section{Discussion}


When populations split and migrate, they may adapt in different ways in response to their local environments. Genetic adaptations that arise and sweep through the population leave a characteristic genomic pattern of long haplotypes of low diversity and high frequency. We develop a two-population haplotype-based statistic, XP-nSL, to capture these patterns. With good power to detect hard and soft sweeps that occur in

491 one population but not another, XP-nSL can identify positively selected regions of the 492 genome likely the result of local adaptation. We apply this statistic to genomes sampled

493 from a pair of wild-born populations of Chinese rhesus macaques, inferred to have 494 diverged approximately 9500 generations ago, one of which lives at high altitude in far western Sichuan province, and the other that lives close to sea level.

497 atmospheric oxygen, increased UV exposure, harsh winters, and reduced nutrition

498 availability, which create strong selection pressure. Organisms that survive and persist 499 are likely to be carrying genetic mutations that confer an advantage for living in such

500 harsh environments. Common targets for adaptation to such an environment include 501 genes related to hypoxia, regulation of ROS, DNA damage repair, and metabolism

502 (Cheviron and Brumfield 2012; Witt and Huerta-Sanchez 2019; Storz and Cheviron 503 2021). Indeed, in the high-altitude macaque population, we identify a strong signal of 504 positive selection at the EGLN1 locus (Fig. 6), a classic target for adaptation to low505 oxygen environments, in addition to 302 other genes, many of which are related to the 506 myriad environmental selection pressures expected in high-altitude environments. As 507 has been suggested previously for other organisms (Cheviron and Brumfield 2012; 508 Bigham and Lee 2014; Simonson 2015; Witt and Huerta-Sanchez 2019; Storz and 
509 Cheviron 2021), these results suggest that, rather than a single adaptive mutation at a

510 single locus, adaptation to this extreme environment by rhesus macaques is polygenic

511 and acts through multiple biological systems.

\section{Acknowledgments}

513 The authors would like to thank members of the Stevison Lab for helpful discussions,

514 Lawrence Uricchio for helpful comments on early versions of the manuscript, and two

515 very helpful anonymous reviewers. This work was supported by start-up funds from the

516 Department of Biological Sciences at Auburn University (LSS) and the Department of

517 Biology at the Pennsylvania State University (ZAS). ZAS was partially supported by

518 NSF-DEB EAGER No. 1939090 (LSS). Portions of this research were performed on the

519 Pennsylvania State University's Institute for Computational Data Sciences' Roar

520 supercomputer.

\section{Author Contributions}

522 ZAS and LSS conceived of the study. ZAS performed all simulations and genomic

523 analyses and implemented novel statistics. ZAS, TEN, and NPB characterized gene

524 functions and ontologies with contributions from LSS. ZAS wrote the manuscript with

525 contributions from NPB, TEN, and LSS. All authors read and approved of the

526 manuscript.

\section{Data Accessibility}

528 Macaque whole genome VCFs are available at http://dx.doi.org/10.5524/100484. Selection scan

529 data available at https://doi.org/10.5061/dryad.kkwh70s40. 


\section{Tables}

533 Table 1. Demographic parameters used for simulations with $95 \%$ confidence intervals 534 from (Liu et al. 2018). T values are given in number of generations before present. $\mathrm{N}$ 535 values represent diploid effective population size.

536

537

538

539

540

541

\begin{tabular}{|c|c|c|}
\hline Parameter & Value & $\mathbf{9 5 \%} \mathbf{~ C l}$ \\
\hline$T_{1}$ & 4660 & $(651,5034)$ \\
\hline$T_{2}$ & 5605 & $(3962,10468)$ \\
\hline$T_{3}$ & 9468 & $(4563,14047)$ \\
\hline$N_{1}$ & 16188 & $(8255,26825)$ \\
\hline$N_{2}$ & 3730 & $(660,5006)$ \\
\hline$N_{3}$ & 4506 & $(572,124135)$ \\
\hline$N_{4}$ & 20768 & $(1710,88242)$ \\
\hline$N_{5}$ & 66210 & $(20581,301430)$ \\
\hline
\end{tabular}

Table 2. Gene ontology enrichment analysis results based on maximum XP-nSL scores per gene. Significant GO terms are enriched for high XP-nSL scores.

\begin{tabular}{|l|l|l|l|}
\hline PANTHER GO-Slim Biological Process & Gene Ontology ID & p-value & FDR \\
\hline anterograde trans-synaptic signaling & $\mathrm{GO} 0098916$ & $4.43 \times 10^{-5}$ & $1.52 \times 10^{-2}$ \\
\hline chemical synaptic transmission & $\mathrm{GO} 0007268$ & $4.43 \times 10^{-5}$ & $1.83 \times 10^{-2}$ \\
\hline regulation of transport & $\mathrm{GO}: 0051049$ & $3.60 \times 10^{-5}$ & $1.85 \times 10^{-2}$ \\
\hline trans-synaptic signaling & $\mathrm{GO}: 0099537$ & $6.32 \times 10^{-5}$ & $1.86 \times 10^{-2}$ \\
\hline synaptic signaling & $\mathrm{GO}: 0099536$ & $1.03 \times 10^{-4}$ & $2.66 \times 10^{-2}$ \\
\hline ion transport & $\mathrm{GO}: 0006811$ & $1.33 \times 10^{-4}$ & $2.75 \times 10^{-2}$ \\
\hline transmembrane transport & $\mathrm{GO}: 0055085$ & $1.57 \times 10^{-4}$ & $2.94 \times 10^{-2}$ \\
\hline regulation of ion transport & $\mathrm{GO}: 0043269$ & $1.30 \times 10^{-4}$ & $2.97 \times 10^{-2}$ \\
\hline metal ion transport & $\mathrm{GO}: 0030001$ & $2.21 \times 10^{-4}$ & $3.04 \times 10^{-2}$ \\
\hline regulation of localization & $\mathrm{GO} 0032879$ & $2.13 \times 10^{-4}$ & $3.14 \times 10^{-2}$ \\
\hline regulation of ion transmembrane transport & $\mathrm{GO}: 0034765$ & $2.66 \times 10^{-4}$ & $3.23 \times 10^{-2}$ \\
\hline inorganic ion transmembrane transport & $\mathrm{GO} 0098660$ & $3.01 \times 10^{-4}$ & $3.45 \times 10^{-2}$ \\
\hline sodium ion transport & $\mathrm{GO}: 0006814$ & $3.36 \times 10^{-4}$ & $3.65 \times 10^{-2}$ \\
\hline ion transmembrane transport & $\mathrm{GO} 0034220$ & $4.50 \times 10^{-4}$ & $4.42 \times 10^{-2}$ \\
\hline regulation of transmembrane transport & $\mathrm{GO}: 0034762$ & $4.40 \times 10^{-4}$ & $4.54 \times 10^{-2}$ \\
\hline cation transport & $\mathrm{GO} 0006812$ & $4.87 \times 10^{-4}$ & $4.57 \times 10^{-2}$ \\
\hline
\end{tabular}

542

543

544

545

546

547

548

549

550

551 
552 Table 3. The top ten $100 \mathrm{~kb}$ genomic regions as ranked by percentage of scores

553 greater than 2. Concatenated region represents the genomic region merged with

554 adjacent top 1\% regions. Max score represents the max XP-nSL score in the

555 concatenated region. Genes gives all genes overlapping the concatenated region.

556

\begin{tabular}{|c|c|c|c|c|c|}
\hline $\begin{array}{l}\text { Genomic } \\
\text { Region }\end{array}$ & $\%>2$ & $\begin{array}{l}\text { Flanking } \\
\text { Regions }\end{array}$ & $\begin{array}{c}\text { Concatenated } \\
\text { Region }\end{array}$ & $\begin{array}{l}\text { Max } \\
\text { Score }\end{array}$ & Genes \\
\hline $\begin{array}{l}{ }^{*} \mathrm{chr} 2: 42300001- \\
42400000\end{array}$ & $81.62 \%$ & 3 & $\begin{array}{l}\text { chr2:42200001- } \\
42600000\end{array}$ & 5.42043 & STXBP5L \\
\hline $\begin{array}{l}\text { chr3:99100001- } \\
99200000\end{array}$ & $78.18 \%$ & 3 & $\begin{array}{l}\text { chr3:99100001- } \\
99500000\end{array}$ & 5.54282 & - \\
\hline $\begin{array}{l}\text { chr1:207600001- } \\
207700000\end{array}$ & $77.39 \%$ & 1 & $\begin{array}{l}\text { chr1:207600001- } \\
207800000\end{array}$ & $6.54809^{t}$ & EGLN1, TSNAX \\
\hline $\begin{array}{l}\text { chr10:69700001- } \\
69800000\end{array}$ & $76.60 \%$ & 4 & $\begin{array}{l}\text { chr10:69700001- } \\
70200000\end{array}$ & 4.89388 & $\begin{array}{l}\text { PITPNB, } \\
\text { ENSMMUT00000079195.1, } \\
\text { TTC28 }\end{array}$ \\
\hline $\begin{array}{l}\text { chr13:64300001- } \\
64400000\end{array}$ & $74.01 \%$ & 1 & $\begin{array}{l}\text { chr13:64300001- } \\
64500000\end{array}$ & 4.17728 & $\begin{array}{l}\text { WDPCP, MDH1, } \\
\text { ENSMMUT00000070474.1 }\end{array}$ \\
\hline $\begin{array}{l}\text { chr7:96400001- } \\
96500000\end{array}$ & $73.86 \%$ & 1 & $\begin{array}{l}\text { chr7:96400001- } \\
96600000\end{array}$ & 4.58886 & $\begin{array}{l}\text { FAM177A1, PPP2R3C, } \\
\text { ENSMMUT00000039911.2 } \\
\text { ENSMMUT00000027928.3 }\end{array}$ \\
\hline $\begin{array}{l}\text { chr10:70400001- } \\
70500000\end{array}$ & $70.24 \%$ & 0 & $\begin{array}{l}\text { chr10:70400001- } \\
70500000\end{array}$ & 3.69192 & TTC28 \\
\hline $\begin{array}{l}{ }^{*} \mathrm{chr} 2: 42400001- \\
42500000\end{array}$ & $65.86 \%$ & 3 & $\begin{array}{l}\text { chr2:42200001- } \\
42600000\end{array}$ & 5.42043 & STXBP5L \\
\hline $\begin{array}{l}\text { chr7:120400001- } \\
120500000\end{array}$ & $62.54 \%$ & 0 & $\begin{array}{l}\text { chr7:120400001- } \\
120500000\end{array}$ & 5.86114 & $\begin{array}{l}\text { KIAA0586, } \\
\text { ENSMMUT00000059161.1 }\end{array}$ \\
\hline $\begin{array}{l}\text { chr18:20600001- } \\
20700000\end{array}$ & $62.42 \%$ & 0 & $\begin{array}{l}\text { chr18:20600001- } \\
20700000\end{array}$ & 5.37386 & RNF138 \\
\hline
\end{tabular}

${ }^{*}$ These regions are contained in the same concatenated region.

tTop genome-wide score. This region contains 6 of the top 10 genome-wide scores.

Table S1. Population classification by individual ID.

561

562

Table S2. List of genomic windows with the top $1 \%$ highest fraction of extreme XP-nSL scores.

Table S3. Estimates of false positive rates for various demographic history normalization scenarios.

\begin{tabular}{|l|c|}
\hline Demographic History & Estimated False Positive Rate \\
\hline Matched & $9.908 \times 10^{-3}$ \\
\hline "Rand" & 0 \\
\hline "Under" & 0 \\
\hline "Over" & 0.9538 \\
\hline
\end{tabular}


573 Table S4. Bin boundaries (\# of sites), number of windows per bin, and top 1\%

574 thresholds for the XP-nSL analysis of rhesus macaques.

\begin{tabular}{|c|c|c|}
\hline $\begin{array}{l}\text { Bin Boundaries (min \# sites, } \\
\text { max \# sites) }\end{array}$ & \# Windows in Bin & $\begin{array}{l}\text { Top 1\% Threshold } \\
\text { (Fraction of Scores }>\text { 2) }\end{array}$ \\
\hline$(11,731)$ & 2672 & 0.346755 \\
\hline$(732,987)$ & 2680 & 0.262945 \\
\hline$(988,1155)$ & 2676 & 0.207089 \\
\hline$(1156,1285)$ & 2675 & 0.196678 \\
\hline$(1286,1390)$ & 2663 & 0.226605 \\
\hline$(1391,1484)$ & 2687 & 0.19274 \\
\hline$(1485,1576)$ & 2665 & 0.163597 \\
\hline$(1577,1681)$ & 2648 & 0.169275 \\
\hline$(1682,1824)$ & 2666 & 0.164025 \\
\hline$(1825,5766)$ & 2666 & 0.153176 \\
\hline
\end{tabular}

575

576

Figures

577

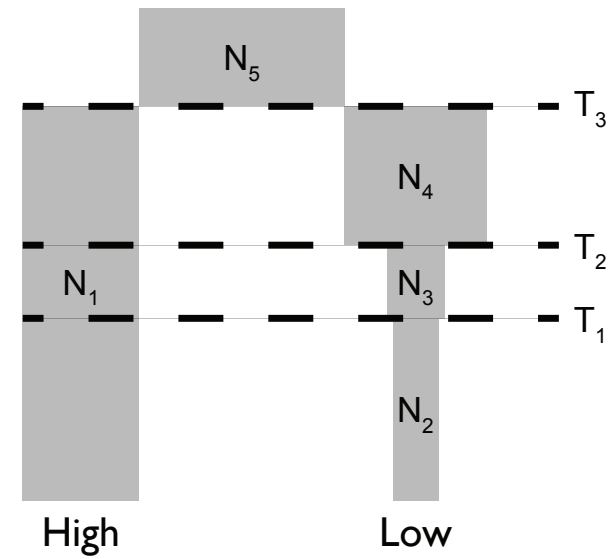

Altitude

Altitude

Figure 1. A representation of the demographic history for our high- and low-altitude populations as inferred by Liu et al. (2018). 


\section{Pop 1}
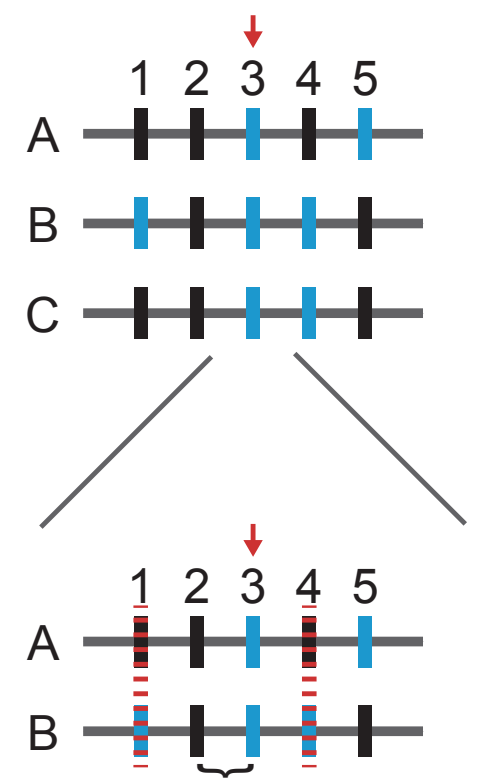

$\mathrm{L}_{\mathrm{AB}}(3)=2$
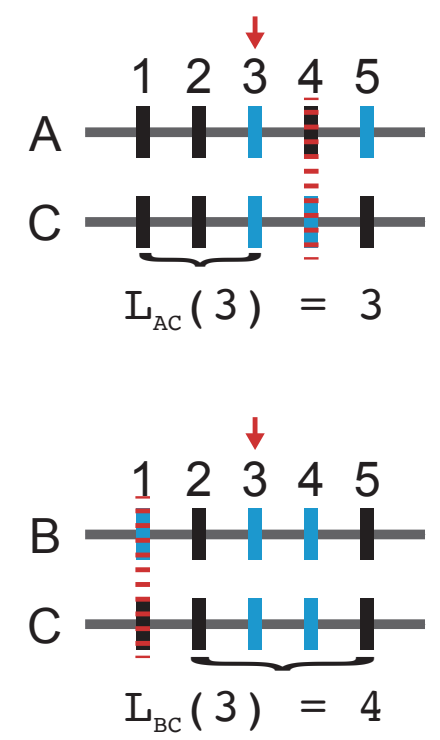

$$
\begin{aligned}
\mathrm{SL}_{\mathrm{P} 1}(3) & =(2+3+4) / 3 \\
& =3
\end{aligned}
$$

Pop 2
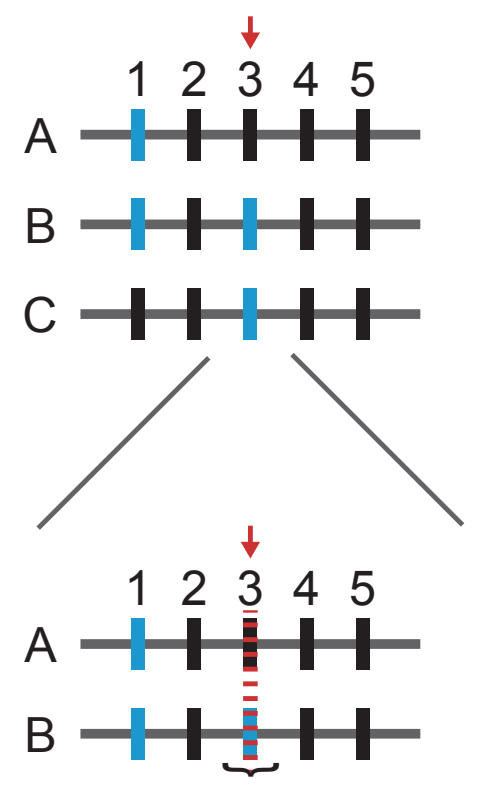

$\mathrm{L}_{\mathrm{AB}}(3)=0$
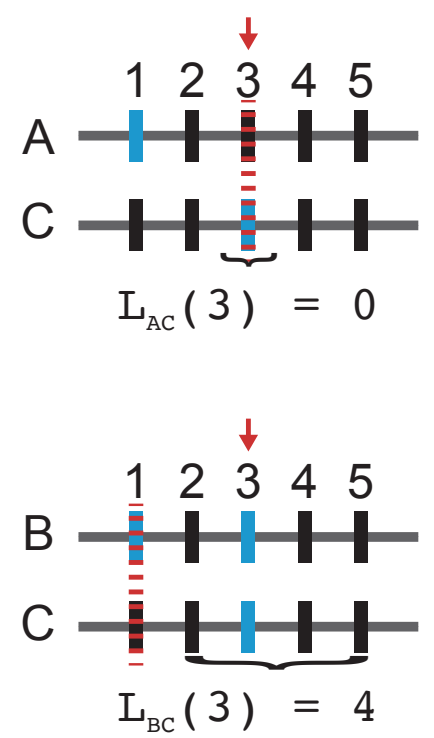

$$
\begin{aligned}
\operatorname{XPnSL}(3) & =\log \left(\operatorname{SL}_{\mathrm{P} 1}(3) / \mathrm{SL}_{\mathrm{P} 2}(3)\right) \\
& =\log (9 / 4)=0.3522
\end{aligned}
$$


589 Figure 2. A toy example illustrating the computation of XP-nSL at a single site in two 590 populations with three haplotypes (grey horizontal bars labeled A-C) and five sites 591 (vertical bars labeled 1-5) where different alleles are colored blue or black. XP-nSL is 592 calculated at site 3 (marked by red arrow). In each population, for each pair of

593 haplotypes, the number of identical-by-state (IBS) sites are counted extending out from 594 and including the test site (red arrow) until reaching a non-IBS site (marked by red 595 dotted line). Within each population, the mean number of IBS sites is calculated across 596 all pairs of haplotypes, and then the log-ratio of the mean from each population is 597 computed to get XP-nSL at site 3. 


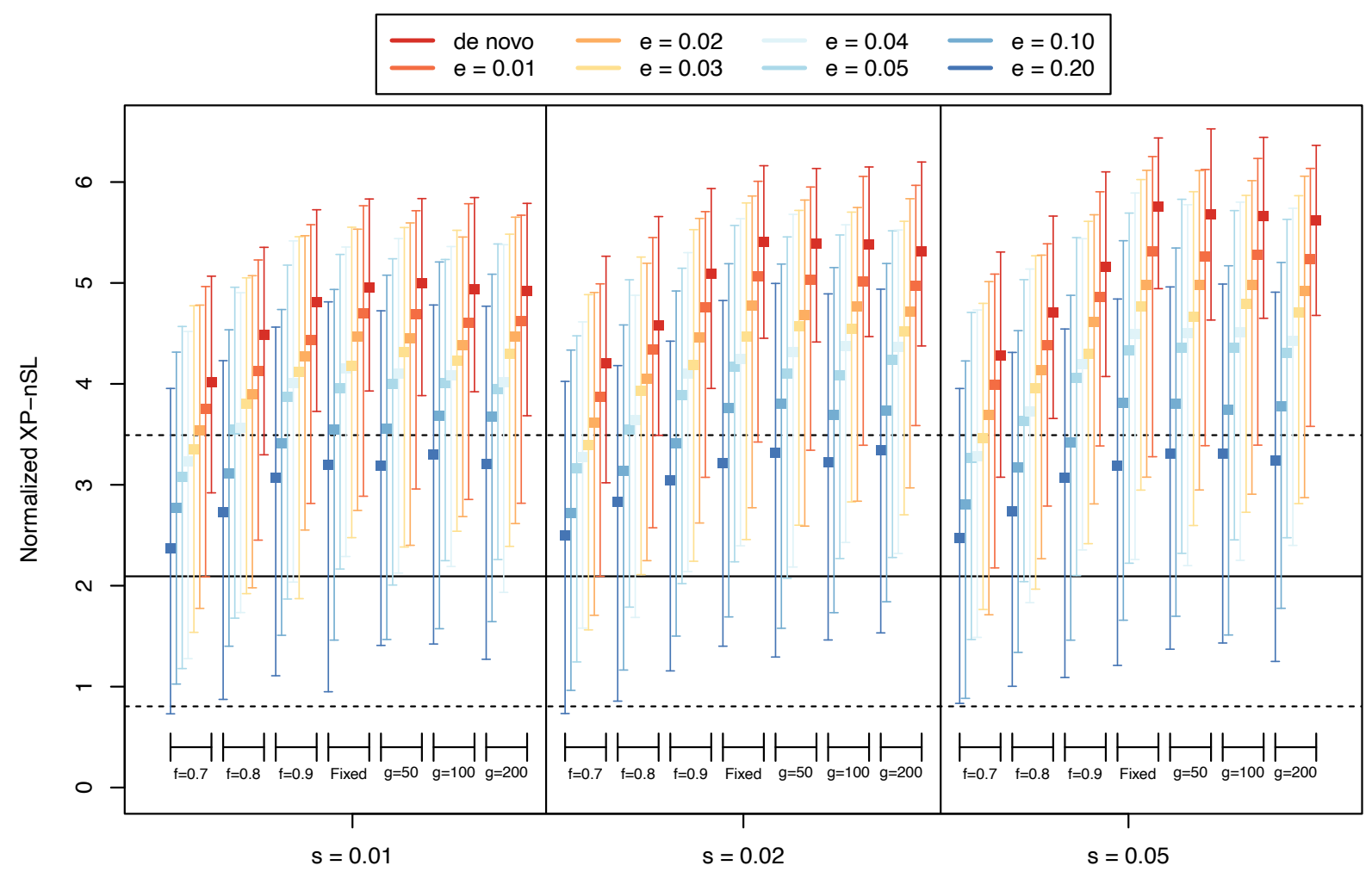

614

Figure 3. The distribution of maximum XP-nSL scores from simulations across various parameters, represented by medians and intervals containing $95 \%$ of the mass of the distribution. Neutral simulations are represented by the black solid horizonal line (median) and the black dashed horizonal line (95\% interval). Non-neutral simulations represented by a colored box (median) and error bars (95\% interval). The parameters are e (frequency at which selection begins, e $>0$ indicates soft sweep), $f$ (frequency of selected mutation at sampling), $g$ (number of generations since fixation), and $\mathrm{s}$ (selection coefficient). 
A

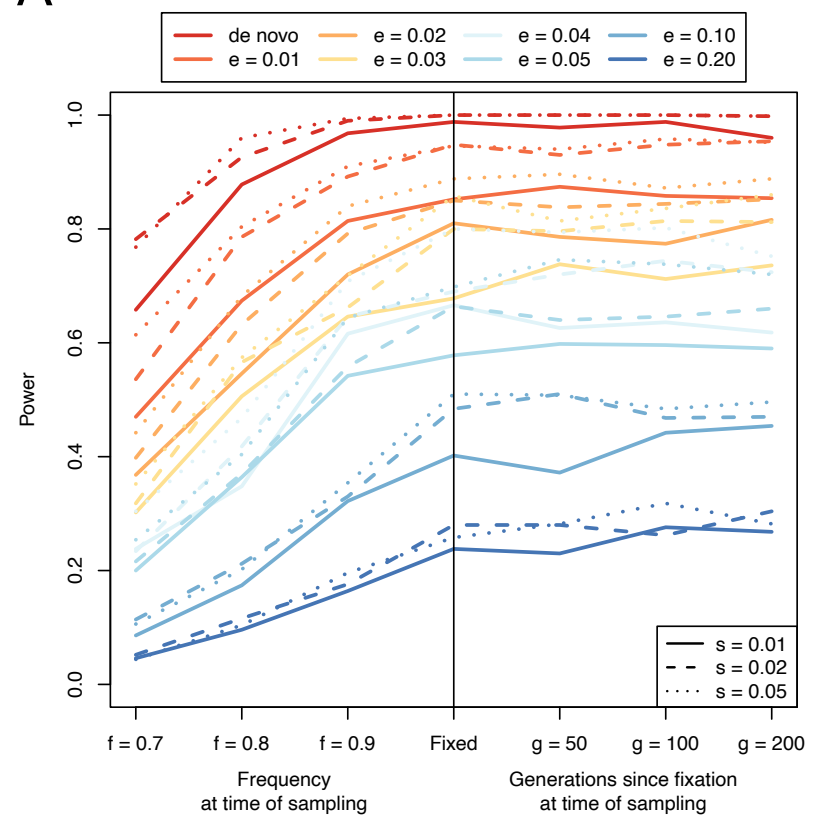

B

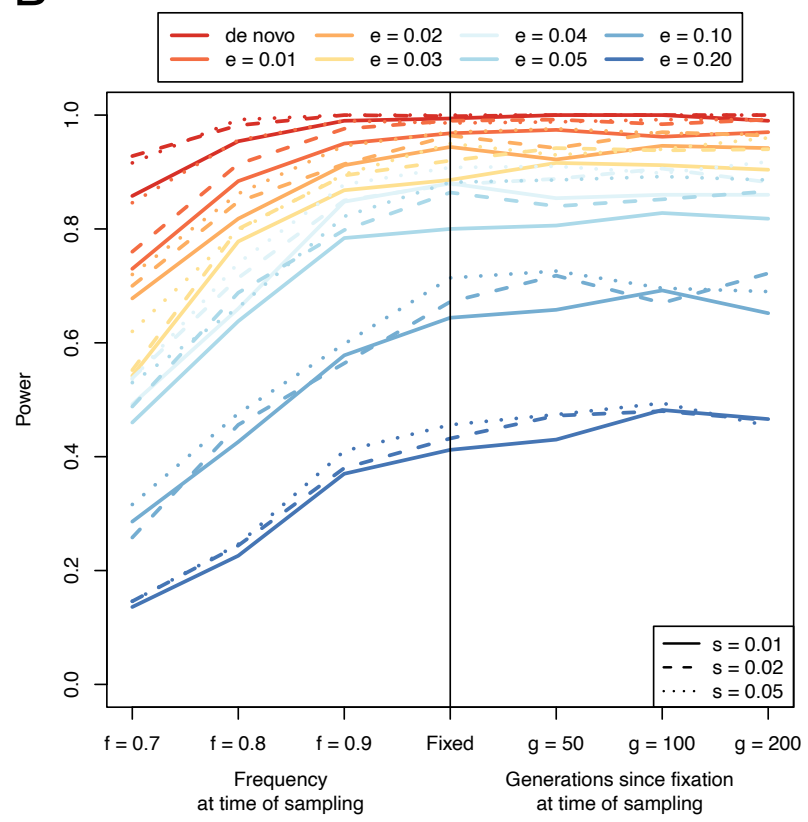

632 Figure 4. Power curves for $(A)$ the max-score approach and $(B)$ the window-based 633 approach to identifying sweeps. The parameters are e (frequency at which selection 634 begins, $e>0$ indicates soft sweep), $f$ (frequency of selected mutation at sampling), $g$ 635 (number of generations since fixation), and $s$ (selection coefficient).

636

637

638

639

640

641

642 
bioRxiv preprint doi: https://doi.org/10.1101/2020.05.19.104380; this version posted February 24, 2021. The copyright holder for this preprint (which was not certified by peer review) is the author/funder, who has granted bioRxiv a license to display the preprint in perpetuity. It is made available under aCC-BY 4.0 International license.

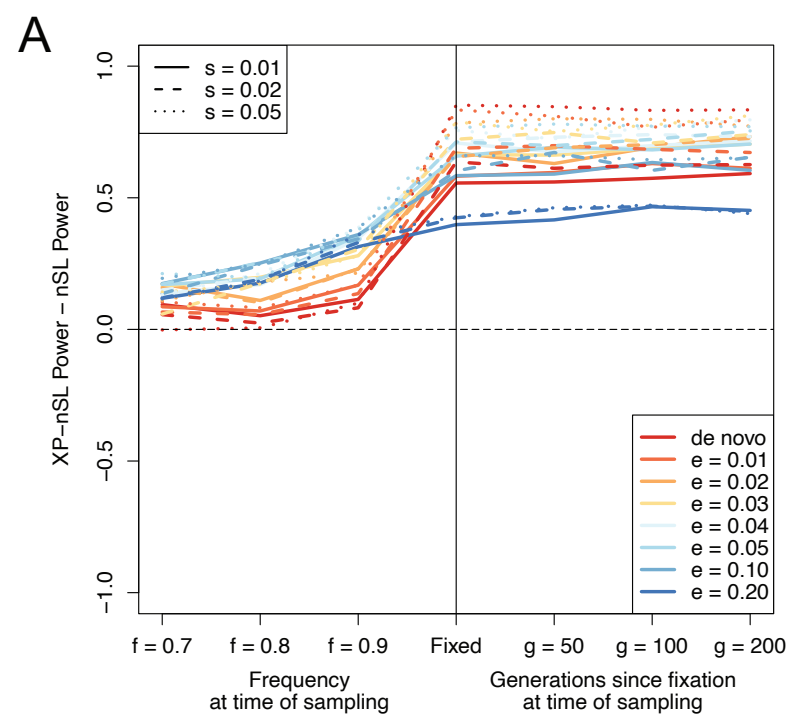

B

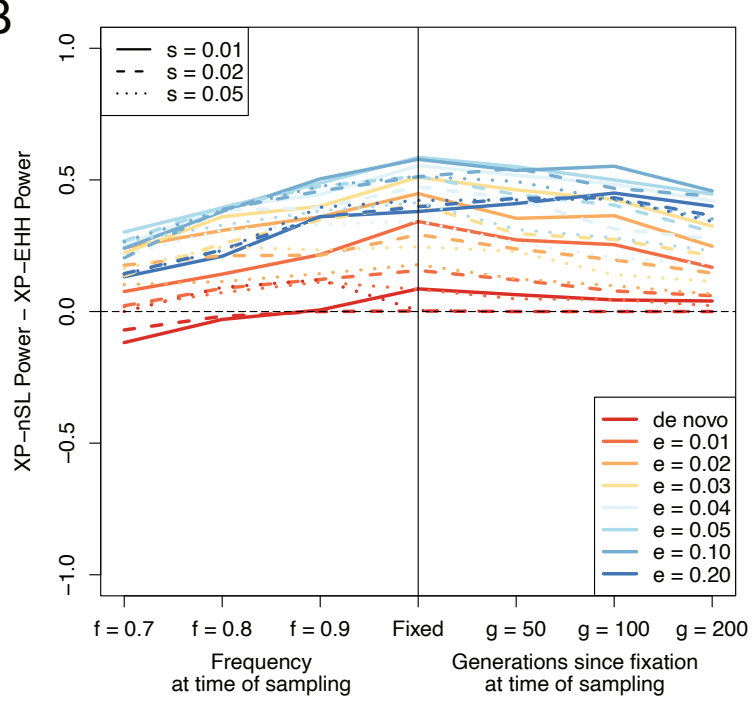

C

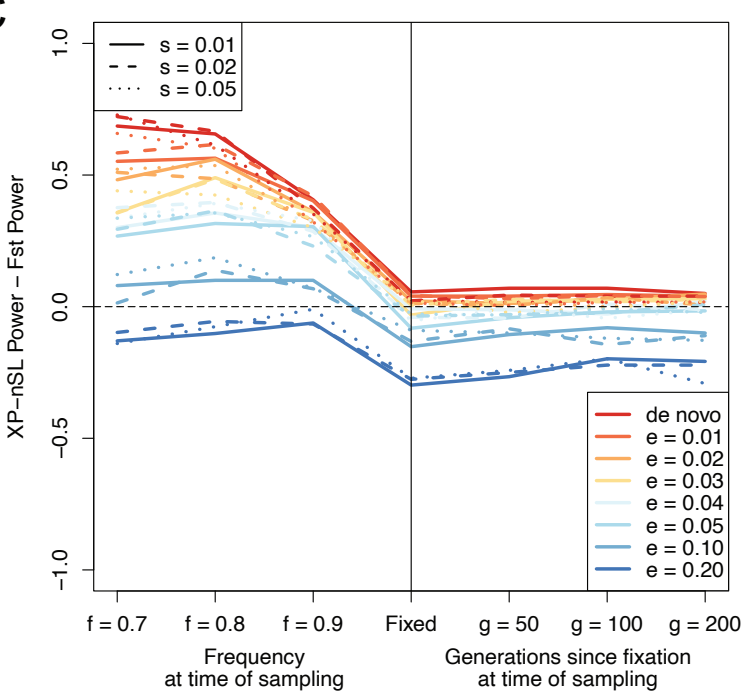


644 Figure 5. Difference in power between XP-nSL and (A) nSL, (B) XP-EHH, and (C) FsT. 645 Values above 0 indicate XP-nSL has more power, and values below 0 indicate XP-nSL 646 has less power. The horizontal black dotted line marks 0 . The parameters are $e$ 647 (frequency at which selection begins, $e>0$ indicates soft sweep), $f$ (frequency of 648 selected mutation at sampling), $g$ (number of generations since fixation), and $\mathrm{s}$ 649 (selection coefficient). 


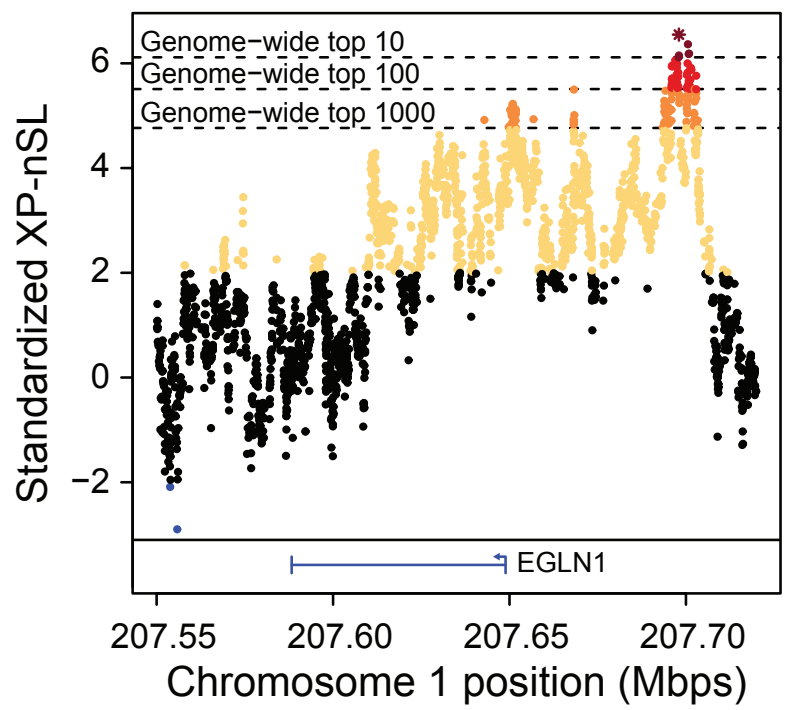

678 Figure 6. XP-nSL scores in the vicinity of the EGLN1 locus. This locus contains the 679 genome wide top score (star) and six of the top ten genome wide scores (dark red).

680

681

682 


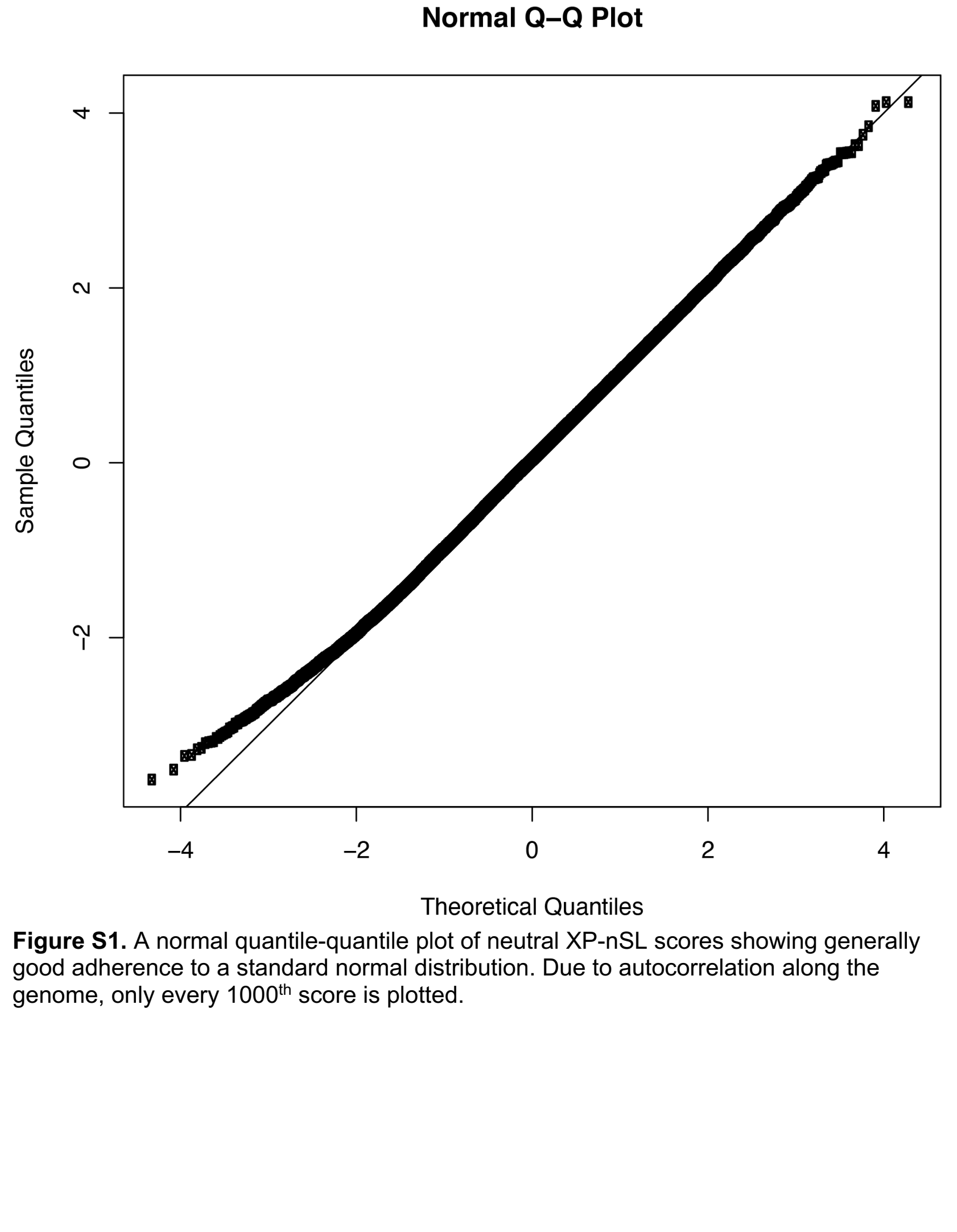

683

684

685

686

687

688

689

690

691

692

693

694

\section{Normal Q-Q Plot}

Theoretical Quantiles good adherence to a standard normal distribution. Due to autocorrelation along the genome, only every $1000^{\text {th }}$ score is plotted. 


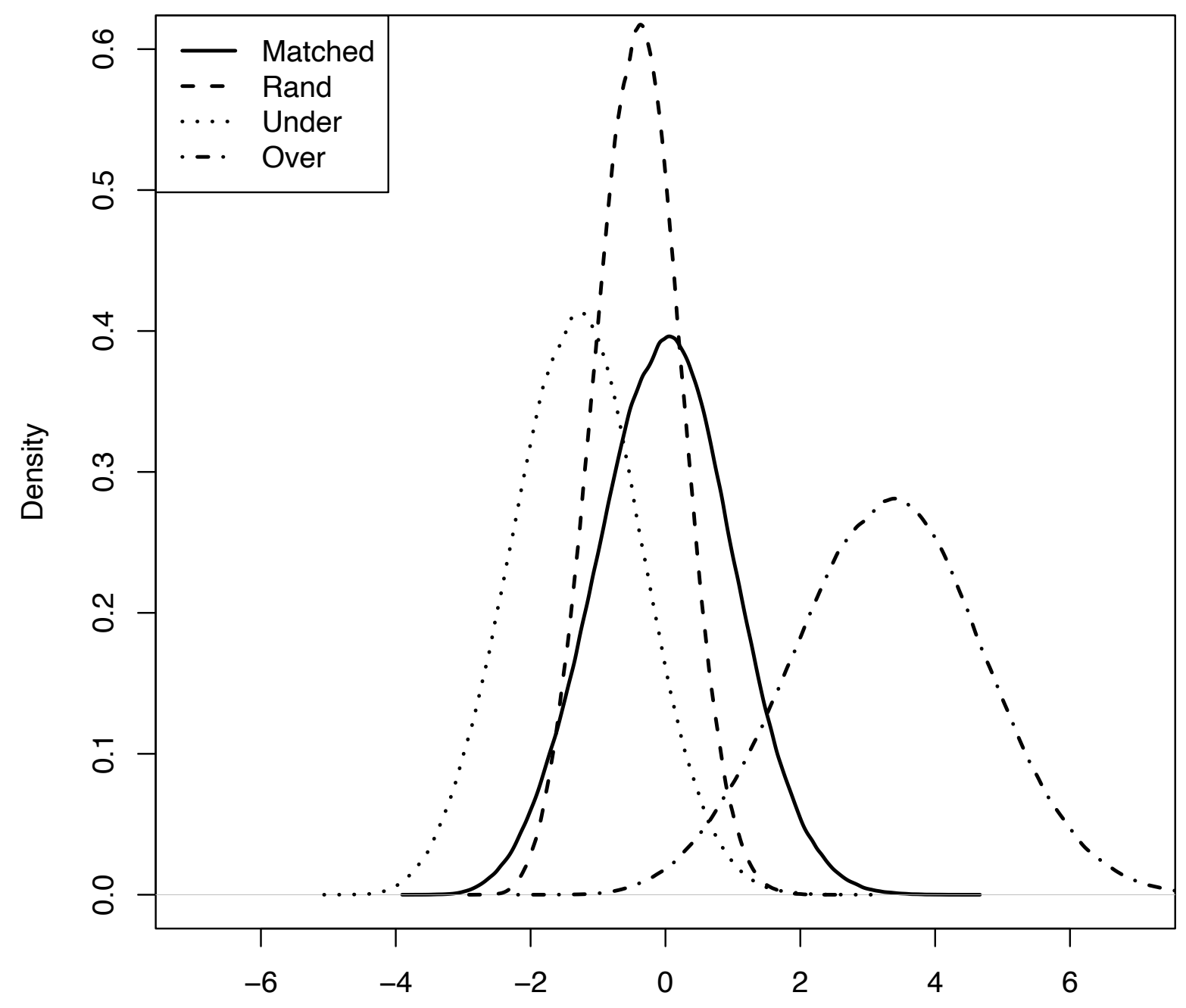

695

Normalized Neutral XP-nSL

Figure S2. The distribution of neutral XP-nSL scores normalized with a matched demographic history (solid line), normalized with the "Rand" demographic history (dashed line), normalized with the "Under" demographic history (dotted line), and normalized with the "Over" demographic history (dash-dot line). Normalizing with the wrong demographic history can dramatically shift the distribution of neutral XP-nSL scores. 
bioRxiv preprint doi: https://doi.org/10.1101/2020.05.19.104380; this version posted February 24, 2021. The copyright holder for this preprint (which was not certified by peer review) is the author/funder, who has granted bioRxiv a license to display the preprint in perpetuity. It is made available under aCC-BY 4.0 International license.
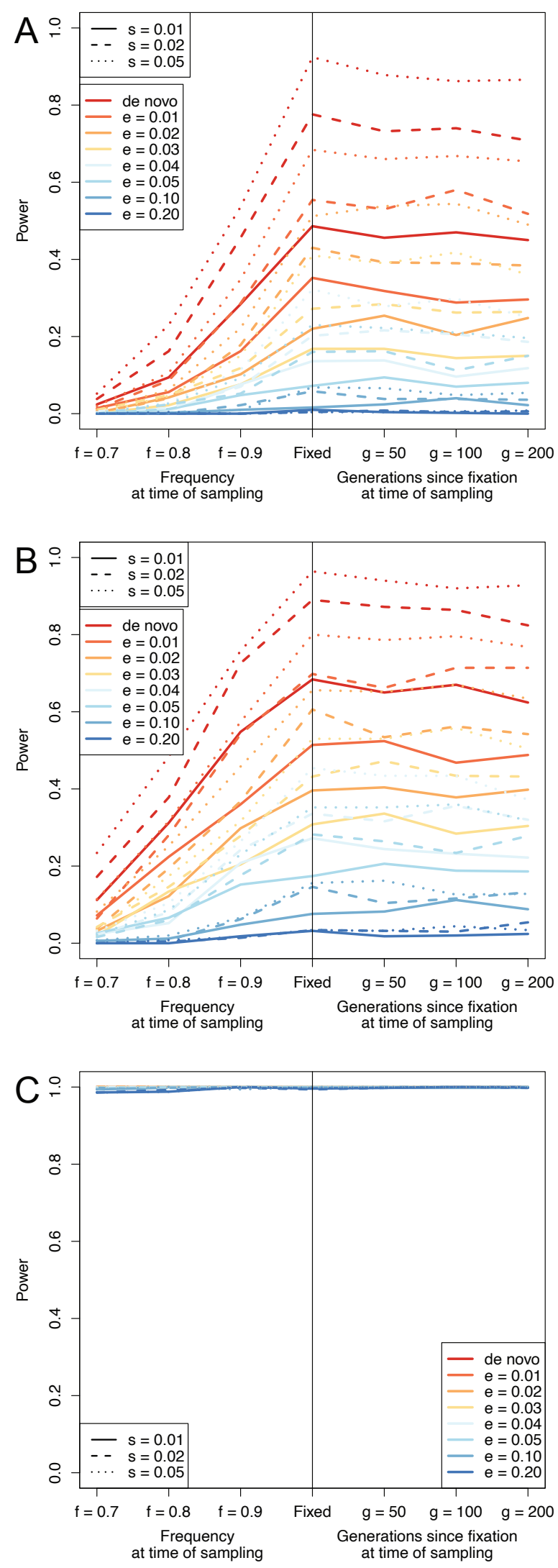
bioRxiv preprint doi: https://doi.org/10.1101/2020.05.19.104380; this version posted February 24, 2021. The copyright holder for this preprint

(which was not certified by peer review) is the author/funder, who has granted bioRxiv a license to display the preprint in perpetuity. It is made available under aCC-BY 4.0 International license.

707 Figure S3. XP-nSL power using mismatched demographic histories for normalization.

708 (A) Using the "Rand" history. (B) Using the "Under" history. (C) Using the "Over" history.

709 
bioRxiv preprint doi: https://doi.org/10.1101/2020.05.19.104380; this version posted February 24, 2021. The copyright holder for this preprint (which was not certified by peer review) is the author/funder, who has granted bioRxiv a license to display the preprint in perpetuity. It is made available under aCC-BY 4.0 International license.
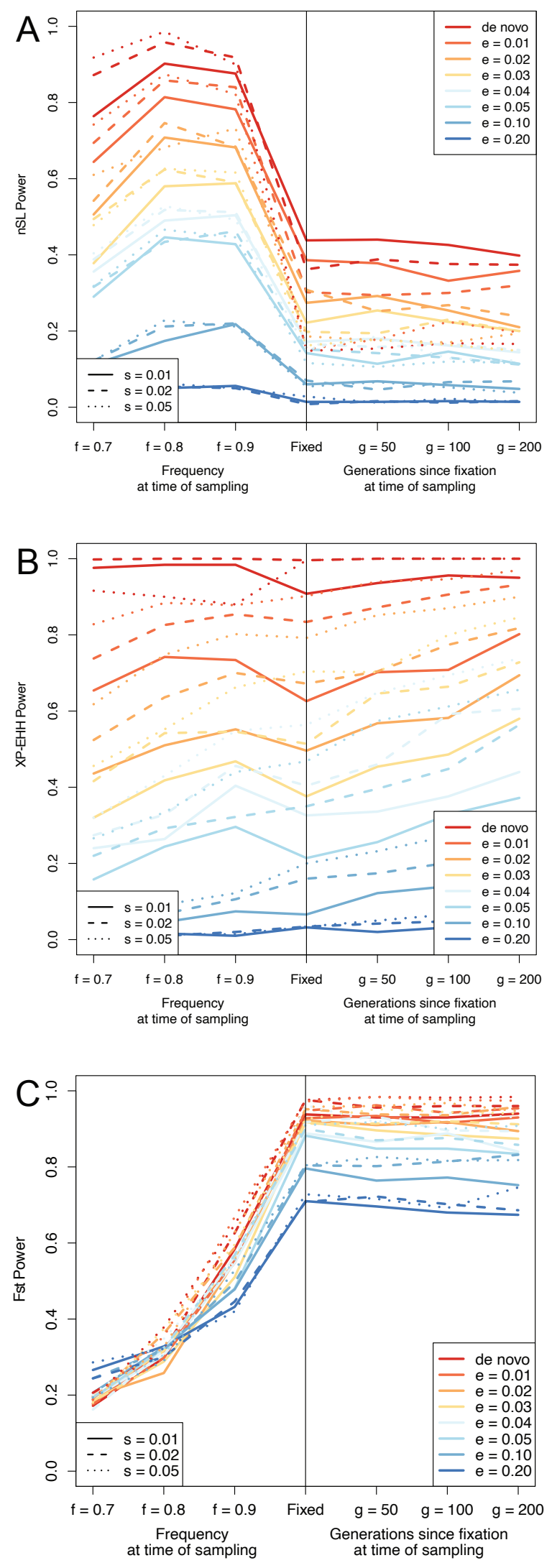
711 Figure S4. Power curves for (A) nSL, (B) XP-EHH, and (C) F $F_{\text {ST. }}$ The parameters are e 712 (frequency at which selection begins, $e>0$ indicates soft sweep), $f$ (frequency of

713 selected mutation at sampling), $g$ (number of generations since fixation), and $\mathrm{s}$

714 (selection coefficient).

715

716

717

718

719

720

721

722

723

724

725

726

727

728 


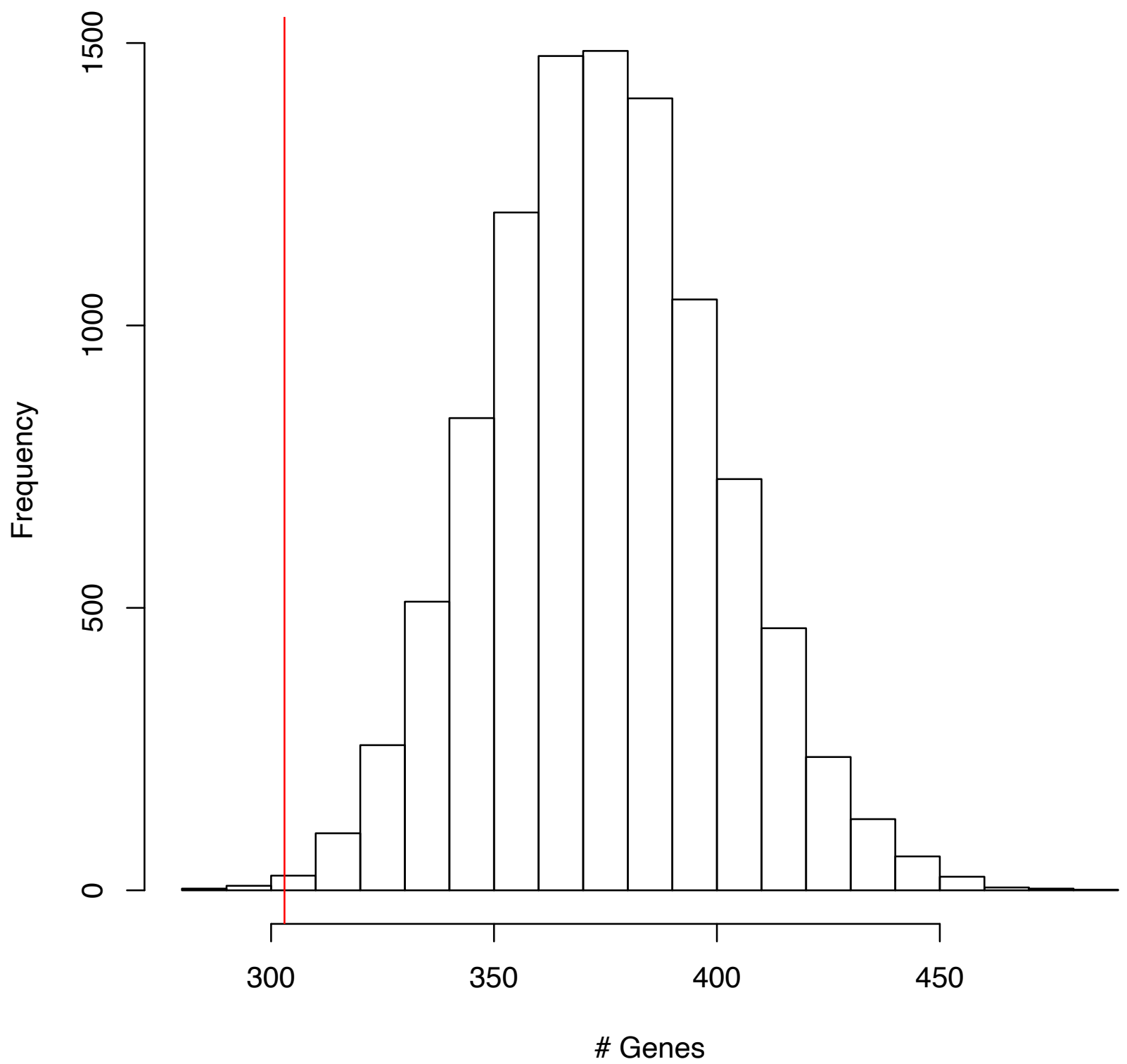

Figure S5. A permutation test (10,000 replicates) that shuffles $270100 \mathrm{~kb}$ regions around the macaque genome and counts the number of unique genes overlapping. The red vertical line marks the 303 genes found in the real data analysis. The probability of observing 303 or fewer genes is $1.4 \times 10^{-3}$, indicating the analysis is not randomly choosing gene regions. 


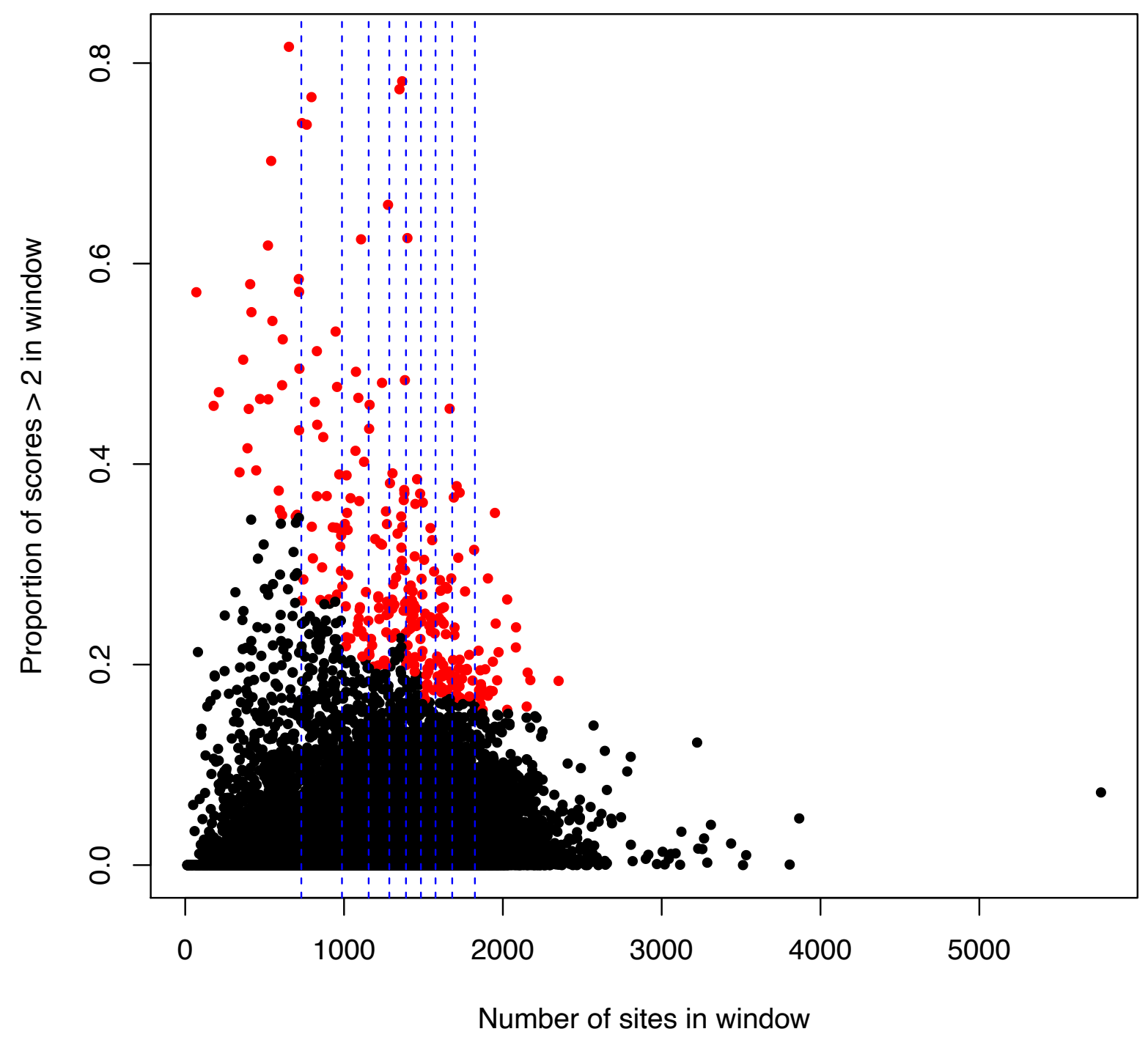

743

744

745

746

747

748

749

750

751

752

753

Figure S6. Proportion of scores $>2$ versus number of sites in window. Blue vertical dashed lines indicate bin boundaries. Each circle is a window, red dots indicate a proportion of scores $>2$ beyond the $1 \%$ threshold for that bin. 


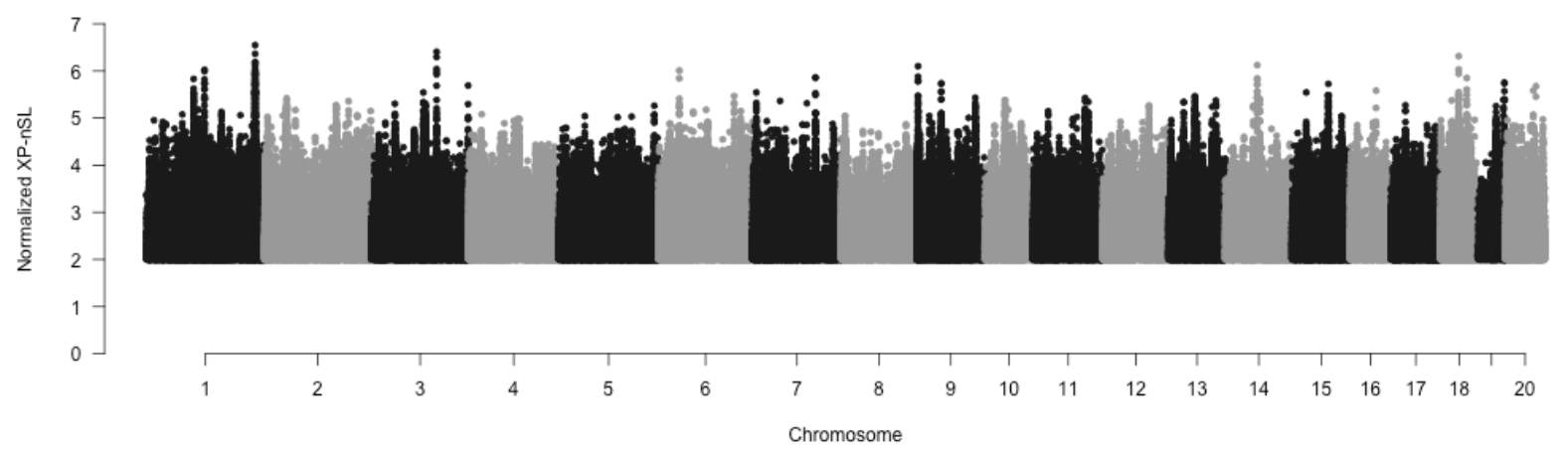

Figure S7. A Manhattan plot of normalized XP-nSL scores across the genome. Due to a very large number of points, only scores $>2$ were plotted. 
790

791

792

793

794

795

796

797

798

799

800

801

802

803

804

805

806

807

808

809

810

811

812

813

814

815

816

817

818

819

820

821

822

823

824

825

826

827

828

829

830

831

832

833

\section{References}

Aarts, M., lihara, K., Wei, W.L., Xiong, Z.G., Arundine, M., Cerwinski, W. et al. (2003). A key role for TRPM7 channels in anoxic neuronal death. Cell 115: 863-877.

Ahmad, K.S., Hameed, M., Fatima, S., Ashraf, M., Ahmad, F., Naseer, M. et al. (2016). Morphoanatomical and physiological adaptations to high altitude in some Aveneae grasses from Neelum Valley, Western Himalayan Kashmir. Acta Physiologiae Plantarum 38: 93.

Alston, C.L., Rocha, M.C., Lax, N.Z., Turnbull, D.M. \& Taylor, R.W. (2017). The genetics and pathology of mitochondrial disease. J Pathol 241: 236-250.

BCM-HGSC. (2020) Baylor College of Medicine Human Genome Sequencing Center Rhemac8 Recombination Map. Available at:

[ftp://ftp.hgsc.bcm.edu/ucscHub/rhesusSNVs/rheMac8/all.rate.bw].

Bigham, A., Bauchet, M., Pinto, D., Mao, X., Akey, J.M., Mei, R. et al. (2010). Identifying signatures of natural selection in Tibetan and Andean populations using dense genome scan data. PLoS Genet 6: e1001116.

Bigham, A.W. \& Lee, F.S. (2014). Human high-altitude adaptation: forward genetics meets the HIF pathway. Genes Dev 28: 2189-2204.

Bigham, A.W., Mao, X., Mei, R., Brutsaert, T., Wilson, M.J., Julian, C.G. et al. (2009). Identifying positive selection candidate loci for high-altitude adaptation in Andean populations. Hum Genomics 4: 79-90.

Cai, Q., Qian, X., Lang, Y., Luo, Y., Xu, J., Pan, S. et al. (2013). Genome sequence of ground tit Pseudopodoces humilis and its adaptation to high altitude. Genome Biol 14: R29.

Campbell, K.L., Storz, J.F., Signore, A.V., Moriyama, H., Catania, K.C., Payson, A.P. et al. (2010). Molecular basis of a novel adaptation to hypoxic-hypercapnia in a strictly fossorial mole. BMC Evol Biol 10: 214.

Cardenas, A., Villalba, A., de Juan Romero, C., Pico, E., Kyrousi, C., Tzika, A.C. et al. (2018). Evolution of Cortical Neurogenesis in Amniotes Controlled by Robo Signaling Levels. Cell 174: 590-606 e521.

Chappell, M.A. \& Hammond, K.A. (2004). Maximal aerobic performance of deer mice in combined cold and exercise challenges. J Comp Physiol B 174: 41-48.

Cheviron, Z.A., Bachman, G.C., Connaty, A.D., McClelland, G.B. \& Storz, J.F. (2012). Regulatory changes contribute to the adaptive enhancement of thermogenic capacity in highaltitude deer mice. Proceedings of the National Academy of Sciences 109: 8635-8640.

Cheviron, Z.A. \& Brumfield, R.T. (2012). Genomic insights into adaptation to high-altitude environments. Heredity 108: 354-361.

Corcoran, A. \& O'Connor, J.J. (2013). Hypoxia-inducible factor signalling mechanisms in the central nervous system. Acta Physiol (Oxf) 208: 298-310.

Danecek, P., Auton, A., Abecasis, G., Albers, C.A., Banks, E., DePristo, M.A. et al. (2011). The variant call format and VCFtools. Bioinformatics 27: 2156-2158.

Delaneau, O., Zagury, J.F., Robinson, M.R., Marchini, J.L. \& Dermitzakis, E.T. (2019). Accurate, scalable and integrative haplotype estimation. Nat Commun 10: 5436.

Dewey, F.E., Gusarova, V., Dunbar, R.L., O'Dushlaine, C., Schurmann, C., Gottesman, O. et al. (2017). Genetic and Pharmacologic Inactivation of ANGPTL3 and Cardiovascular Disease. N Engl J Med 377: 211-221. 
Fan, Z., Zhou, A., Osada, N., Yu, J., Jiang, J., Li, P. et al. (2018). Ancient hybridization and admixture in macaques (genus Macaca) inferred from whole genome sequences. $\mathrm{Mol}$ Phylogenet Evol 127: 376-386.

Ferrer-Admetlla, A., Liang, M., Korneliussen, T. \& Nielsen, R. (2014). On detecting incomplete soft or hard selective sweeps using haplotype structure. Mol Biol Evol 31: 1275-1291.

Fooden, J. (2000). Systematic review of the rhesus macaque, Macaca mulatta (Zimmermann, 1780)Field Museum of Natural History, Chicago, III. :.

Foti, A., Dorendorf, F. \& Leimkuhler, S. (2017). A single nucleotide polymorphism causes enhanced radical oxygen species production by human aldehyde oxidase. PLoS One 12: e0182061.

Garud, N.R., Messer, P.W., Buzbas, E.O. \& Petrov, D.A. (2015). Recent selective sweeps in North American Drosophila melanogaster show signatures of soft sweeps. PLoS Genet 11: e1005004.

Ge, R.-L., Cai, Q., Shen, Y.-Y., San, A., Ma, L., Zhang, Y. et al. (2013). Draft genome sequence of the Tibetan antelope. Nature Communications 4: 1858.

Gonzalo-Turpin, H. \& Hazard, L. (2009). Local adaptation occurs along altitudinal gradient despite the existence of gene flow in the alpine plant species Festuca eskia. Journal of Ecology 97: 742-751.

Graham, A.M. \& McCracken, K.G. (2019). Convergent evolution on the hypoxia-inducible factor (HIF) pathway genes EGLN1 and EPAS1 in high-altitude ducks. Heredity (Edinb) 122: 819832.

Greinert, R., Volkmer, B., Henning, S., Breitbart, E.W., Greulich, K.O., Cardoso, M.C. et al. (2012). UVA-induced DNA double-strand breaks result from the repair of clustered oxidative DNA damages. Nucleic Acids Res 40: 10263-10273.

Guo, T., Yin, R.X., Huang, F., Yao, L.M., Lin, W.X. \& Pan, S.L. (2016). Association between the DOCK7, PCSK9 and GALNT2 Gene Polymorphisms and Serum Lipid levels. Sci Rep 6: 19079.

Guo, X., Hu, Q., Hao, G., Wang, X., Zhang, D., Ma, T. et al. (2018). The genomes of two Eutrema species provide insight into plant adaptation to high altitudes. DNA Research 25: 307315.

Hayes, J.P. \& Chappell, M.A. (1986). Effects of Cold Acclimation on Maximum Oxygen Consumption during Cold Exposure and Treadmill Exercise in Deer Mice, Peromyscus maniculatus. Physiological Zoology 59: 473-481.

Hebbar, P., Nizam, R., Melhem, M., Alkayal, F., Elkum, N., John, S.E. et al. (2018). Genome-wide association study identifies novel recessive genetic variants for high TGs in an Arab population. J Lipid Res 59: 1951-1966.

Hendrickson, S.L. (2013). A genome wide study of genetic adaptation to high altitude in feral Andean Horses of the paramo. BMC Evol Biol 13: 273.

Hermisson, J. \& Pennings, P.S. (2005). Soft sweeps: molecular population genetics of adaptation from standing genetic variation. Genetics 169: 2335-2352.

Hernandez, R.D., Hubisz, M.J., Wheeler, D.A., Smith, D.G., Ferguson, B., Rogers, J. et al. (2007). Demographic histories and patterns of linkage disequilibrium in Chinese and Indian rhesus macaques. Science 316: 240-243. 
Huerta-Sanchez, E., Degiorgio, M., Pagani, L., Tarekegn, A., Ekong, R., Antao, T. et al. (2013). Genetic signatures reveal high-altitude adaptation in a set of ethiopian populations. $\mathrm{Mol}$ Biol Evol 30: 1877-1888.

Huerta-Sanchez, E., Jin, X., Asan, Bianba, Z., Peter, B.M., Vinckenbosch, N. et al. (2014). Altitude adaptation in Tibetans caused by introgression of Denisovan-like DNA. Nature 512: 194197.

Ismail, I.H., Gagne, J.P., Genois, M.M., Strickfaden, H., McDonald, D., Xu, Z. et al. (2015). The RNF138 E3 ligase displaces Ku to promote DNA end resection and regulate DNA repair pathway choice. Nat Cell Biol 17: 1446-1457.

Jandl, K., Thekkekara Puthenparampil, H., Marsh, L.M., Hoffmann, J., Wilhelm, J., Veith, C. et al. (2019). Long non-coding RNAs influence the transcriptome in pulmonary arterial hypertension: the role of PAXIP1-AS1. J Pathol 247: 357-370.

Janocha, A.J., Comhair, S.A.A., Basnyat, B., Neupane, M., Gebremedhin, A., Khan, A. et al. (2017). Antioxidant defense and oxidative damage vary widely among high-altitude residents. Am J Hum Biol 29.

Jeong, C., Alkorta-Aranburu, G., Basnyat, B., Neupane, M., Witonsky, D.B., Pritchard, J.K. et al. (2014). Admixture facilitates genetic adaptations to high altitude in Tibet. Nat Commun 5: 3281.

Karle, C., Gehrig, T., Wodopia, R., Hoschele, S., Kreye, V.A., Katus, H.A. et al. (2004). Hypoxiainduced inhibition of whole cell membrane currents and ion transport of A549 cells. Am J Physiol Lung Cell Mol Physiol 286: L1154-1160.

Kern, A.D. \& Schrider, D.R. (2016). Discoal: flexible coalescent simulations with selection. Bioinformatics 32: 3839-3841.

Kim, E. \& Donohue, K. (2013). Local adaptation and plasticity of Erysimum capitatum to altitude: its implications for responses to climate change. Journal of Ecology 101: 796-805.

Kim, Y. \& Nielsen, R. (2004). Linkage Disequilibrium as a Signature of Selective Sweeps. Genetics 167: 1513-1524.

Krueger, S.K. \& Williams, D.E. (2005). Mammalian flavin-containing monooxygenases: structure/function, genetic polymorphisms and role in drug metabolism. Pharmacol Ther 106: 357-387.

Kumar, R., Corbett, M.A., Smith, N.J., Jolly, L.A., Tan, C., Keating, D.J. et al. (2015). Homozygous mutation of STXBP5L explains an autosomal recessive infantile-onset neurodegenerative disorder. Hum Mol Genet 24: 2000-2010.

Li, M., Tian, S., Jin, L., Zhou, G., Li, Y., Zhang, Y. et al. (2013). Genomic analyses identify distinct patterns of selection in domesticated pigs and Tibetan wild boars. Nat Genet 45: 14311438.

Li, Y., Wu, D.D., Boyko, A.R., Wang, G.D., Wu, S.F., Irwin, D.M. et al. (2014). Population variation revealed high-altitude adaptation of Tibetan mastiffs. Mol Biol Evol 31: 1200-1205.

Liu, J.-Q., Duan, Y.-W., Hao, G., Ge, X.-J. \& Sun, H. (2014). Evolutionary history and underlying adaptation of alpine plants on the Qinghai-Tibet Plateau. Journal of Systematics and Evolution 52: 241-249.

Liu, Z., Tan, X., Orozco-terWengel, P., Zhou, X., Zhang, L., Tian, S. et al. (2018). Population genomics of wild Chinese rhesus macaques reveals a dynamic demographic history and local adaptation, with implications for biomedical research. Gigascience 7. 
Madrid, J.E., Mandalaywala, T.M., Coyne, S.P., Ahloy-Dallaire, J., Garner, J.P., Barr, C.S. et al. (2018). Adaptive developmental plasticity in rhesus macaques: the serotonin transporter gene interacts with maternal care to affect juvenile social behaviour. Proc Biol Sci 285.

Mi, H., Muruganujan, A., Ebert, D., Huang, X. \& Thomas, P.D. (2019). PANTHER version 14: more genomes, a new PANTHER GO-slim and improvements in enrichment analysis tools. Nucleic Acids Res 47: D419-D426.

Munne-Bosch, S., Cotado, A., Morales, M., Fleta-Soriano, E., Villellas, J. \& Garcia, M.B. (2016). Adaptation of the Long-Lived Monocarpic Perennial Saxifraga longifolia to High Altitude. Plant Physiol 172: 765-775.

O'Reilly, P.F., Birney, E. \& Balding, D.J. (2008). Confounding between recombination and selection, and the Ped/Pop method for detecting selection. Genome Res 18: 1304-1313.

Osada, N. \& Akashi, H. (2012). Mitochondrial-nuclear interactions and accelerated compensatory evolution: evidence from the primate cytochrome $\mathrm{C}$ oxidase complex. Mol Biol Evol 29: 337-346.

Peng, Y., Yang, Z., Zhang, H., Cui, C., Qi, X., Luo, X. et al. (2011). Genetic variations in Tibetan populations and high-altitude adaptation at the Himalayas. Mol Biol Evol 28: 1075-1081.

Pennings, P.S. \& Hermisson, J. (2006). Soft sweeps II--molecular population genetics of adaptation from recurrent mutation or migration. Mol Biol Evol 23: 1076-1084.

Przeworski, M. (2002). The Signature of Positive Selection at Randomly Chosen Loci. Genetics 160: 1179-1189.

Qiu, Q., Zhang, G., Ma, T., Qian, W., Wang, J., Ye, Z. et al. (2012). The yak genome and adaptation to life at high altitude. Nat Genet 44: 946-949.

Qu, Y., Zhao, H., Han, N., Zhou, G., Song, G., Gao, B. et al. (2013). Ground tit genome reveals avian adaptation to living at high altitudes in the Tibetan plateau. Nat Commun 4: 2071.

Ramasamy, S.K., Kusumbe, A.P., Wang, L. \& Adams, R.H. (2014). Endothelial Notch activity promotes angiogenesis and osteogenesis in bone. Nature 507: 376-380.

Richard, A.F., Goldstein, S.J. \& Dewar, R.E. (1989). Weed macaques: The evolutionary implications of macaque feeding ecology. International Journal of Primatology 10: 569.

Rodriguez, J., Pilkington, R., Garcia Munoz, A., Nguyen, L.K., Rauch, N., Kennedy, S. et al. (2016). Substrate-Trapped Interactors of PHD3 and FIH Cluster in Distinct Signaling Pathways. Cell Rep 14: 2745-2760.

Rosenmann, M. \& Morrison, P. (1974). Maximum oxygen consumption and heat loss facilitation in small homeotherms by He-O2. Am J Physiol 226: 490-495.

Sabeti, P.C., Reich, D.E., Higgins, J.M., Levine, H.Z., Richter, D.J., Schaffner, S.F. et al. (2002). Detecting recent positive selection in the human genome from haplotype structure. Nature 419: 832-837.

Sabeti, P.C., Varilly, P., Fry, B., Lohmueller, J., Hostetter, E., Cotsapas, C. et al. (2007). Genomewide detection and characterization of positive selection in human populations. Nature 449: 913-918.

Schweizer, R.M., Velotta, J.P., Ivy, C.M., Jones, M.R., Muir, S.M., Bradburd, G.S. et al. (2019). Physiological and genomic evidence that selection on the transcription factor Epas 1 has altered cardiovascular function in high-altitude deer mice. PLoS Genet 15: e1008420. 
Shang, P., Li, W., Liu, G., Zhang, J., Li, M., Wu, L. et al. (2019). Identification of IncRNAs and Genes Responsible for Fatness and Fatty Acid Composition Traits between the Tibetan and Yorkshire Pigs. Int J Genomics 2019: 5070975.

Simonson, T.S. (2015). Altitude Adaptation: A Glimpse Through Various Lenses. High Alt Med Biol 16: 125-137.

Stewart, C.B. \& Disotell, T.R. (1998). Primate evolution - in and out of Africa. Curr Biol 8: R582588.

Storz, J.F. \& Cheviron, Z.A. (2021). Physiological Genomics of Adaptation to High-Altitude Hypoxia. Annu Rev Anim Biosci 9: 149-171.

Storz, J.F., Cheviron, Z.A., McClelland, G.B. \& Scott, G.R. (2019). Evolution of physiological performance capacities and environmental adaptation: insights from high-elevation deer mice (Peromyscus maniculatus). J Mammal 100: 910-922.

Storz, J.F., Sabatino, S.J., Hoffmann, F.G., Gering, E.J., Moriyama, H., Ferrand, N. et al. (2007). The molecular basis of high-altitude adaptation in deer mice. PLoS Genet 3: e45.

Szpiech, Z.A. \& Hernandez, R.D. (2014). selscan: an efficient multithreaded program to perform EHH-based scans for positive selection. Mol Biol Evol 31: 2824-2827.

Tanaka, T., Inazawa, J. \& Nakamura, Y. (1996). Molecular cloning and mapping of a human cDNA for cytosolic malate dehydrogenase (MDH1). Genomics 32: 128-130.

To, K.K. \& Huang, L.E. (2005). Suppression of hypoxia-inducible factor 1alpha (HIF-1alpha) transcriptional activity by the HIF prolyl hydroxylase EGLN1. J Biol Chem 280: 3810238107.

Velotta, J.P., Robertson, C.E., Schweizer, R.M., McClelland, G.B. \& Cheviron, Z.A. (2020). Adaptive shifts in gene regulation underlie a developmental delay in thermogenesis in high-altitude deer mice. Molecular Biology and Evolution.

Voight, B.F., Kudaravalli, S., Wen, X. \& Pritchard, J.K. (2006). A map of recent positive selection in the human genome. Plos Biol 4: e72.

Wang, G.D., Fan, R.X., Zhai, W., Liu, F., Wang, L., Zhong, L. et al. (2014). Genetic convergence in the adaptation of dogs and humans to the high-altitude environment of the tibetan plateau. Genome Biol Evol 6: 2122-2128.

Wang, M.-S., Li, Y., Peng, M.-S., Zhong, L., Wang, Z.-J., Li, Q.-Y. et al. (2015). Genomic Analyses Reveal Potential Independent Adaptation to High Altitude in Tibetan Chickens. Molecular Biology and Evolution 32: 1880-1889.

Wang, M.-S., Wang, S., Li, Y., Jhala, Y., Thakur, M., Otecko, N.O. et al. (2020). Ancient hybridization with an unknown population facilitated high altitude adaptation of canids. Molecular Biology and Evolution.

Wang, X., Takenaka, K. \& Takeda, S. (2010). PTIP promotes DNA double-strand break repair through homologous recombination. Genes Cells 15: 243-254.

Weir, B.S. \& Cockerham, C.C. (1984). Estimating F-Statistics for the Analysis of Population Structure. Evolution 38: 1358-1370.

Witt, K.E. \& Huerta-Sanchez, E. (2019). Convergent evolution in human and domesticate adaptation to high-altitude environments. Philos Trans R Soc Lond B Biol Sci 374: 20180235. 
1006

1007

1008

1009

1010

1011

1012

1013

1014

1015

1016

1017

1018

1019

1020

1021

1022

1023

1024

1025
Xing, J., Wang, M., Hong, J., Gao, Y., Liu, Y., Gu, H. et al. (2019). TRPM7 channel inhibition exacerbates pulmonary arterial hypertension through MEK/ERK pathway. Aging (Albany NY) 11: 4050-4065.

Xu, S., Li, S., Yang, Y., Tan, J., Lou, H., Jin, W. et al. (2010). A Genome-Wide Search for Signals of High-Altitude Adaptation in Tibetans. Molecular Biology and Evolution 28: 1003-1011.

Xu, Z., Jin, X., Cai, W., Zhou, M., Shao, P., Yang, Z. et al. (2018). Proteomics Analysis Reveals Abnormal Electron Transport and Excessive Oxidative Stress Cause Mitochondrial Dysfunction in Placental Tissues of Early-Onset Preeclampsia. Proteomics Clin Appl 12: e1700165.

Yang, X., Wang, Y., Zhang, Y., Lee, W.H. \& Zhang, Y. (2016). Rich diversity and potency of skin antioxidant peptides revealed a novel molecular basis for high-altitude adaptation of amphibians. Sci Rep 6: 19866.

Ye, B., Hou, N., Xiao, L., Xu, Y., Xu, H. \& Li, F. (2016). Dynamic monitoring of oxidative DNA double-strand break and repair in cardiomyocytes. Cardiovasc Pathol 25: 93-100.

Yi, X., Liang, Y., Huerta-Sanchez, E., Jin, X., Cuo, Z.X., Pool, J.E. et al. (2010). Sequencing of 50 human exomes reveals adaptation to high altitude. Science 329: 75-78.

Zhang, Y., Yuan, F., Wu, X., Rechkoblit, O., Taylor, J.S., Geacintov, N.E. et al. (2000). Error-prone lesion bypass by human DNA polymerase eta. Nucleic Acids Res 28: 4717-4724. 